\title{
II Kirchlicher Diskurs und soziale Realität
}

Eine der Grundfragen dieser Arbeit beschäftigt sich mit der Kontinuität der Spiele in der Spätantike. Dass der Aspekt „Kontinuität“ überhaupt als ein besonderer Gegenstand der Diskussion in Erscheinung tritt, wird vor dem Hintergrund der Position kirchlicher Autoritäten verständlich. So besteht ein Großteil des Quellenmaterials aus Predigten oder Traktaten von Klerikern, welche in der gesamten Spätantike eine massive Polemik gegen das traditionelle Spielewesen hervorbrachten. Diese Verurteilungen der heidnischen und unmoralischen Spiele nehmen ihren Anfang in der berühmten Schrift Tertullians de spectaculis an der Wende vom 2. zum 3. Jahrhundert, ${ }^{1}$ setzen sich die Spätantike hindurch fort - sowohl im Westen als auch im Osten - und finden schließlich als argumentativer Topos Einzug in das Mittelalter, ${ }^{2}$ als Johannes von Salisbury im 12. Jahrhundert noch anhand antiker Beispiele einen Text gegen Bühnendarbietungen verfasste. ${ }^{3}$ Auf den ersten Blick mag man einen unveränderbaren Diskurs der Dichotomie zwischen kirchlicher Zugehörigkeit und der Teilnahme an Spielen erkennen, dessen Bestandteile austauschbar waren und $\mathrm{zu}$ verschiedenen Zeiten wiederholt wurden. ${ }^{4}$ Die permanente Kritik der Kirchenväter bezeugt im Umkehrschluss allerdings auch die kontinuierliche Existenz von Spielen und damit zu-

1 Für eine extensive Analyse dieses Werkes siehe LugARESI (2008) 377-427, der deutlich macht, dass bei Tertullian bereits die meisten Themen der späteren Kritik angelegt waren. Allerdings sind auch zuvor bei Clemens von Alexandrien und Tatian Vorbehalte gegenüber paganen Festen zu konstatieren; ebenso verurteilte Iustin die Themen heidnischer Poeten, wie man sie später beim Pantomimus kritisierte: Clem. prot. 3; Tat. orat. 22; Iust. apol 1.21.2-5, 25.1-2 (ed. Minns-Parvis 2009). Eine Übersicht der vortertullianischen Kritik findet sich bei LugARESI (2008) 261-319 u. 463-534. Tertullians Ablehnung der Spiele fußte also ihrerseits auf einem bereits existierenden Diskurs, jedoch ist seine Schrift das erste Dokument, das sich aus christlicher Perspektive ausführlich mit dem römischen Spielewesen auseinandersetzte. Ähnlich danach auch Min. Fel. 12.5-6 u. 37.11-12; Ps. Cypr. [Nov.] spect. (= CSEL 3.3, 3-13); Cypr. ad Donat. 7-8. Zu dem vorkonstantinischen Diskurs allgemein siehe SALLManN (1990) 246.

2 Alcuin epist. 189 (791 n. Chr.) beruft sich auf Augustinus; vgl. auch Conc. Aquisgr. a. $816 \S 83$ u. 100 (= MANSI 14, 202 u. 210); Conc. Paris. a. $829 \S 38$ (= MANSI 14, 562); Bern. Clarav. serm. de conv. 14 (= PL 182, 842) (11. Jdt.);. JÜRGENS (1972) 250.

3 Joh. Salis. polycrat. 7-8 (= PL 199, 405); dazu Chambers (1954) 230. Für das Mittelalter siehe ALLARDYCE (1963) 146-159; Goody (1997) 109-110; ORTALLi (2007/2008). Selbst im 17. Jdt. berief man sich noch auf Tertullian, um Elemente der Volkskultur zu bekämpfen, dazu BuRKE (1981) 231.

4 In Anlehnung an Michael Foucault, jedoch nicht in exakter Nachfolge, soll unter dem Begriff „Diskurs“ in den folgenden Abschnitten dieser Arbeit ein zunächst sprachlich formuliertes, aber auch durch soziale und kulturelle Praktiken hervorgebrachtes Konzept bestimmter Ideen verstanden werden, das sich dem Historiker anhand einer Reihe von Einzelzeugnissen präsentiert, vgl. LANDWEHR (2009) 68-71 u. 102-103. Im Fall der Kirchenväter bestand der Diskurs in der Schaffung einer dichotomischen Beziehung zwischen christlicher Identität und traditionellem Spielewesen. Der Begriff „Diskurs“ erscheint hier angebracht, da er auf den ersten Blick die drei von Foucault aufgestellten Ordnungsmechanismen eines Machtdiskurses erfüllt: externe Ausschließung, interne Kontrolle, Verknappung der sprechenden Subjekte. Es gilt aber zu überprüfen, ob diese literarisch formulierte Ordnung auch in gesellschaftliches Handeln übertragen werden konnte. 
gleich das überwiegende Scheitern der eigenen Strategien bei der Christianisierung sozialer Handlungen. ${ }^{5}$

Die im ersten Teil dieses Kapitels gesammelten Argumente vermitteln einen anschaulichen Eindruck von dem Spektrum an Kritik, welches die christlichen Autoritäten gegenüber den Spielen vorbrachten. Die diskursiven Strategien bewegten sich dabei auf verschiedenen argumentativen Ebenen und umfassten religiöse, moralische, kulturelle wie auch ökonomische Aspekte. Hierbei lassen sich zwar je nach Spielgattung gewisse Schwerpunkte ausmachen, jedoch richtete sich die kirchliche Kritik gegen Wagenrennen, Theaterschauspiele oder Arenakämpfe gleichermaßen und größere Unterschiede sind nicht erkennbar. ${ }^{6} \mathrm{Da}$ das Verhältnis der patristischen Autoren zu den Spielen bereits Gegenstand zahlreicher Studien geworden ist, ${ }^{7}$ wird diese Beschreibung nur in kursorischer Weise vorgenommen. Große Teile der bisherigen Forschung haben allerdings die Position Tertullians oft mit Texten des 4. oder 5. Jahrhunderts verbunden, ohne dabei die chronologische oder inhaltliche Dimension genauer zu beachten. Sicherlich hat die Fundamentalkritik Tertullians alle nachfolgenden Autoren entscheidend beeinflusst, ${ }^{8}$ dennoch fand in späterer Zeit eine deutliche Verlagerung von religiösen zu moralischen Argumenten statt.

Bei der Frage nach einer Kontinuität geht es aber vielmehr darum, im Sinne Richard Lims die polemisch und rhetorisch aufgeladenen Texte über ihre jeweiligen „discursive frames“ hinaus zu betrachten ${ }^{9}$ und zu versuchen, als Historiker die in ihnen enthaltenen Informationen in ein ausgewogenes Verhältnis zur sozialen Realität zu setzen. Es gilt nach den Gründen zu fragen, die in der Praxis zu einem Scheitern der kirchlichen Argumentation über einen Zeitraum von mehr als drei Jahrhunderten geführt haben. Daher liegt der Schwerpunkt im zweiten Teil dieses Kapitels auf einer Analyse der Ambivalenzen und Brüche innerhalb des patristischen Diskurses - anders gesagt auf den inhärenten Faktoren, die einen Erfolg der eigenen Strategien verhinderten. Des Weiteren werden auch externe bzw. übergeordnete Faktoren diskutiert wie der grundsätzliche Gegensatz zwischen asketischer Sichtweise und spätantiker Stadtkultur.

5 So ist es u.a. bezeichnend, dass Minucius Felix eine Abwesenheit seiner Leserschaft bei den Spielen konstruierte, was jedoch kaum mehr als eine literarische Fiktion war (Min. Fel. 12).

6 Vgl. auch LugARESI (1998) 53.

7 Alès (1910); Eriau (1914); Reyval (1924); Waszink (1947); Vandenberghe (1955); Magoulias (1971); Dumortier (1972); Weismann (1972); JÜRgens (1972); PASQuato (1976); HaLPORN (1976); Cramer (1980); Harl (1981); Schnusenberg (1981); MAtTer (1990); Cuscito (1994); Lugaresi (1998); LeYERLE (2001); LugARESI (2003b); RETZLEFF (2003b); Dox (2004); LAFER (2005); KAHLOS (2005); JiMÉNEZ SÁNCHEZ (2006b); LUGARESI (2007); GRAF (2008); JiMÉNEZ SÁNCHEZ (2010) 269-316; zuletzt auch CAMERon (2011) 790-791 und DAGRon (2011) 253-273. Den wohl detailliertesten Überblick über die einzelnen Konfliktfelder bietet die Publikation von LUGARESI (2008); eine kompakte Übersicht findet sich u.a. bei WEBB (2008) 168-171.

8 Vgl. LugaRESI (2005).

9 Lim (2009) 502. Die Mittel hierfür liegen in einer Analyse des Kontexts und einem Vergleich mit anderen zeitgenössischen Quellen (idem 506-507). 
Der auf der literarischen Ebene konstruierte Diskurs einer Dichotomie zwischen christlichem Lebenswandel und der Welt der spectacula konnte nur mit geringem Erfolg in eine soziale Realität umgesetzt werden. Daher wird das Ergebnis sowohl eine Stereotypie und Lebensferne der kirchlichen Argumentation wie auch zahlreiche Überschneidungen der beiden Lebenswelten „Kirche“ und „Spiele“ aufzeigen.

\section{Offizieller Diskurs}

\section{Religiöse Ebene}

Das früheste Argument, um eigene Gläubige von einem Besuch der Spiele abzuhalten, findet sich bei Tertullian, der den Ursprung der Spiele detailliert aus dem heidnischen Kult herleitete und eine Beteiligung daher aufgrund religiöser Kritierien untersagte: ${ }^{10}$ Christliche Zuschauer würden sich durch einen Besuch an paganen Praktiken beteiligen und ihre Teilnahme sei dem Götzendienst gleichzusetzen. ${ }^{11}$

Ein ähnlich religiöser Zusammenhang findet sich auch in der Argumentation späterer Autoren wieder, ${ }^{12}$ wobei man insbesondere auf die Anlässe und Begleitumstände von Spielveranstaltungen verwies: So würden immer noch Praktiken wie zum Beispiel das Anlegen besonderer Gewänder oder Prozessionen gepflegt, die einen heidnischen Ursprung besäßen. ${ }^{13}$ Ebenso seien die Anlässe auf einen ehemals paganen Festkalender zurückzuführen und würden auf nicht-christlichen Bräuchen basieren. ${ }^{14}$

Autoren des 4., 5. und 6. Jahrhunderts führten ihre Kritik zudem nicht mehr nur auf den Ursprung der Spiele zurück, sondern brachten religiöse Argumente auch gegen den paganen Inhalt von Theaterdarbietungen vor: Die Darstellung antiker Mythen durch Pantomimen oder ihre parodistische Inszenierung im Mimus führe dem Zuschauer weiterhin heidnische Göttergestalten vor Augen und sei daher als Blasphemie zu meiden. ${ }^{15}$ Basilius von Seleucia drückte es in der Mitte des 5. Jahrhunderts folgendermaßen aus: ${ }^{16}$

10 Tert. spect. 5-8; $10-13$.

11 Vgl. die Analyse dieser Position bei Schnusenberg (1981) 29-34.

12 Const. apost. 2.62.1-3 (ed. Metzger 1985, SC 320); Jac. Ser. hom. de spect. 3 (= Moss (1935) 105106); Sev. Ant. hom. cat. 54 (= PO 4.1, 48); Salv. gub. 6.11(60-61).

13 Jac. Ser. hom. de spect. 3 (= Moss (1935) 105-106); Jos. Styl. chron. 30 (ed. engl. Trombley - Watt 2000); Sev. Ant. hom. cat. 95 (= PO 25.1, 93-94); vgl. Lib. Or. 1.230.

14 Selbst in justinianischer Zeit spricht Johannes von Gaza z. B. in Bezug auf die Feste seine Hei-

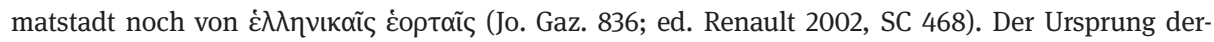
artiger Veranstaltungen lag wahrscheinlich in der Kaiserzeit, worin für Johannes ihr paganer Charakter bestand. Siehe auch MAcMulLEN (1997) 39-45.

15 Aug. civ. 7.26; Bas. Sel. or. 27.1 (= PG 85, 309-312); Chrys. exp. in Ps. 81 (= PG 55, 106); Chrys. hom. 5 in Tit. 4 (= PG 62, 693); Jac. Ser. hom. de spect. 5 (= Moss (1935) 108-112); Sev. Ant. hom. cat. 95 (= PO 25.1, 94-95); GREATREX-WATT (1999) 8-17. Das Grundpoblem lag in der Verbindung solcher 
„Wenn du dir nämlich das anschaust, was man am wenigstens anschauen darf, in welche Reihe soll Christus dich dann wohl einordnen - die der Heiden? Oder soll er dich unter die Gläubigen zählen? [...] So lasst uns nicht Anhänger des Teufels werden, indem wir ihn mit dämonischen Vergnügungen verehren!“

\section{Moralische Vorbehalte}

Die wohl am häufigsten vorgebrachten Argumente waren aber auf moralischer Ebene angesiedelt. Diese Kritik zielte zunächst auf die Zuschauer ab, deren Leidenschaften sich bei Spielveranstaltungen hemmungslos dem Geschehen hingäben und darüber die moralischen Ansprüche an einen Christen vergessen ließen. So wurde das Geschehen bei Wagenrennen im Zirkus vor allem mit dem Motiv ungezügelter Emotionen in Zusammenhang gebracht: Wenn die Zuschauer wie toll agierten und in ihrer Begeisterung für Wagenlenker und Teams sowohl Gott als auch die Kirche vergäßen, dann führe dies nur zu Unruhen und stelle eine moralische Sünde gegenüber den wahren Prioritäten eines christlichen Lebens dar. ${ }^{17}$ In diesem Zusammenhang kritisierten christliche Schriften die bei Spielen auftretenden Unruhen und aggressiven Aussschreitungen der Zirkusparteien als Resultat der Zügellosigkeit von Emotionen. ${ }^{18}$ Damit entsprachen sie den Einschätzungen zeitgenössischer nicht-theologischer Autoren, die ebenfalls das Unruhepotenzial gewaltbereiter Parteianhänger und ihre Bedrohung für das Staatswesen hervorhoben. ${ }^{19}$ Ereignisse im Amphitheater wurden solange die Gladiatorenkämpfe im 4. Jahrhundert noch relevant waren - zwar in moralischer Hinsicht kritisiert, jedoch zumeist nicht aufgrund des Schicksals der Akteure in der Arena, also der Tötung von Menschen. Vielmehr richteten sich die hauptsächlichen Einwände gegen die finanzielle Förderung dieser Spektakel und gegen den Effekt auf die Seelen der Zuschauer, in denen ein Blutrausch verursacht

Geschichten zum griechischen Polytheismus, vgl. Ps.-Just. coh. ad Gr. 15.1-17.1, 36.4 (ed. Riedweg 1994); Jac. Ser. hom. de spect. 3 (= Moss (1935) 105-106); CRAMER (1980) 103-104.

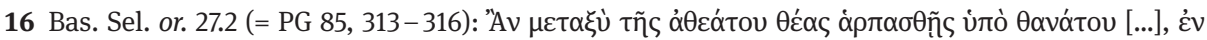

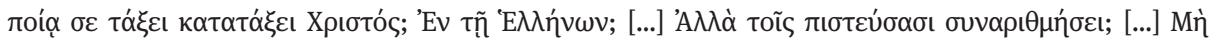

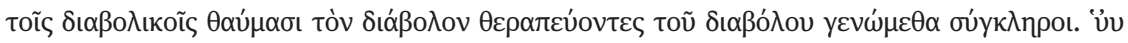

17 Aug. serm. Dolb. inedit. D.8 (= DolbeAU (1996) 63); Jo. D. parall. tit. 31 (= PG 96. 310-312); Salv. gub. 6.3(15) u. 6.4(20); Sev. Ant. hom. cat. 26 (= PO 36.4, 551); hom. cat. 54 (= PO 4.1, 52-54). Zur speziellen Kritik an magischen Praktiken im Zusammenhang mit dem Zirkus siehe HeINTZ (1999) 50 51.

18 Aug. catech. rud. 16.25 (= CC 40, 149-150); Aug. serm. Den. 14.3 (ed. Morin, Misc. Agost. 1930 I); Amph. Seleuc. 150 -180; Bas. Sel. or. 27.1 (= PG 85, 308-313); Doctr. Jac. 1.40, 5.20 (ed. DaGRoNDÉroche (1991)); Isid. Pel. epp. 5.185(1469); Sev. Ant. hom. cat. 26 (= PO 36.4, 551-553).

19 Z. B. Agath. 5.14.4; Menas dial. scient. pol. 5.103-114 (ed. Mazzucchi 1982); Procop. Pers. 24.1-6; ebenso Cassiod. var. 3.51.3. Auch das historiographische Werk von Johannes Malalas weist zahlreiche Berichte über die verheerenden Auswirkungen der Rivalität zwischen den Zirkusparteien auf. Zu dieser Thematik siehe Fotiou (1978); Whitby (1998); Weвв (2008) 169; Meier (2007) 232-233. 
würde..$^{20}$ Eine Verbindung zu den Amphitheatern als ehemalige Stätten des Martyriums wurde bei dieser Kritik bemerkenswerterweise nie gezogen.

Eine in den Augen der Kirchenväter noch größere moralische Gefahr stellten die szenischen Schauspiele dar. Die im Mimus übliche Aufführung von sexuellen Geschichten und Ehebruch und die damit verbundene gelegentliche Entblößung von Schauspielerinnen waren Aspekte, die in inhaltlicher und visueller Hinsicht einen moralischen Schaden für den christlichen Zuschauer bedeuteten: ${ }^{21}$ Durch die Augen und Ohren würden derartige Obszönitäten einen Ehemann zum Ehebruch verführen; zudem diene das Betrachten halbnackter Schauspielerinnen letztlich nur der eigenen Lüsternheit in Gedanken und Taten. ${ }^{22}$ In diesem Sinne war auch der Besuch pantomimischer Darbietungen verwerflich, da hierbei männliche Darsteller als Frauen agierten und so durch ihre Bewegungen Perversionen der Natur hervorbrächten und das Gemüt der Zuschauer verweichlichten. ${ }^{23}$

Gerade das letzte Argument richtete sich nicht nur an den Zuschauer, sondern auch an die Bühnendarsteller selbst: Als Männer handelten sie wider die eigene Natur, wenn sie feminine Bewegungen vollzögen ${ }^{24}$ oder sich ihrem Alter ungemäß aufführten und dadurch ein unsittliches Schauspiel darböten. ${ }^{25}$ Schauspielerinnen würden sich wiederum wie Huren aufführen, wenn sie sich auf der Bühne entblößten. Auch die im Theater übliche Musik und das Tanzen entspreche nicht christlichen Maßstäben und sei eine dämonische Verführung, die durch die Sinne in die Seele eindringen könne. ${ }^{26}$

20 Kritik an Gladiatorenkämpfen ist in den Schriften christlicher Autoren aus der Spätantike selten, da diese im 4. Jdt. bereits im Niedergang begriffen waren, vgl. Ambr. off. 2.21.109 (= PL 16, 140); Paul. Nol. epist. 13.15-16; Prud. c. Symm. 1.114; Eus. v. C. 4.25.1; Hier. vita Hilar. 3.11; Augustinus-Lexikon, Vol. 1, 1986-1994, 301. Zu den hauptsächlichen Kritikpunkten siehe VILLE (1960) 291-294; WIEDEMANN (1992) 147-155; CARLÀ (2010) 290-296. Für weitere Studien zu diesem Thema vgl. Anm. 3 Kapitel VI.

21 Chrys. hom. in I Cor. 7:2 1 (= PG 51, 209).

22 Salv. gub. 6.4.(20); Chrys. de in. glor. 38, 56, 78 (ed. Malingrey 1972, SC 188); Chrys. David 31 (= PG 54, 695-696); Chrys. theatr. 2-3 (= PG 56, 266-268); Chrys. hom. 6 in Mt. 7-8 (= PG 57, 71-72); Chrys. hom. 7 in Mt. 6 (= PG 57, 79-81); Chrys. hom. 5 in 1 Thess. 4 (= PG 62, 427-428); Jac. Ser. hom. de spect. 5 (= Moss (1935)108-112). Vgl. Chor. Apol. mim. 34-35; Sev. Ant. hom. cat. 54 (= PO 4.1, 56); ebenso schon bei Clem. prot. 3. Zur Bedeutung des „sündigen Betrachtens“ siehe WEBB (2008) 182183.

23 Cypr. ad Donat. 8; Sev. Ant. hom. cat. 54 (= PO 4.1, 54); Isid. Etym. 18.53; Chrys. pan. Barl. 4 (= PG 50, 681-682); Chrys. hom. 18 in Jo. 4 (= PG 59, 119-120). Gleiches galt für Scherze: Chrys. hom. 17 in Ephes. 3 (= PG 62, 120); Chrys. hom. 15 in Heb. 4 (= PG 63, 121-124); Sev. Ant. hom. cat. 54 (= PO 4.1, 54); hymn. 269 (= Allen-HAYWARd (2004) 172-73); Jac. Ed. hymn. Sev. Ant. 269 (= PO 7.5, 304-305); WEBB (2008) 186-187.

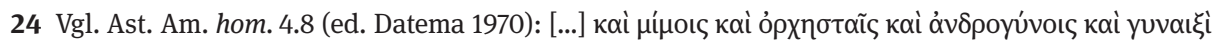
rópvaıc [...]. Ebenso Amph. Seleuc. 90 -99 (ed. Oberg 1969).

25 Cypr. epist. 2; Chrys. subintr. 9 (= PG 47, 508); Chrys. hom. in I Cor. 7:2 2 (= PG 51, 211); Chrys. hom. 37 in Mt. 6 (= PG 57, 426-27); Chrys. hom. 18 in Jo. 4 (= PG 59, 119-120); Isid. Pel. epp. 5.185 (ed. Évieux 2000, SC 454); Conc. Brac. a. 572 frg. $§ 80$ (= MANSI 9, 844); vgl. WeBB (2008) 190.

26 Vgl. Clem. paed. 2.40.2; Clem. strom. 6.90.1-2 (ed. Descourtieux 1999, SC 446); Ambr. in Luc. 7.237 (= CSEL 32, 388); Chrys. exp. in Ps. 81 (= PG 55, 106); Chrys. hom. 11 in 1 Thess. 3 (= PG 62, 466); vgl. 
Zudem existierte eine generelle Verurteilung von theatralischer mimesis, indem die szenische Darstellung als Heuchlerei und Lüge verurteilt wurde. ${ }^{27}$ Ruth Webb hat dargelegt, dass diese Haltung auf einer Ablehnung alles Fiktiven basierte und eine solche Negierung des Mimetischen wiederum die Bühnenakteure in eine „constant oscillation between the true and the false“ stellte. ${ }^{28}$ Die Handlungen weiblicher und männlicher Schauspieler auf der Bühne, insbesondere im Mimus, wurden auf den Charakter des Darstellers selbst übertragen, ${ }^{29}$ den man als unmoralisch und provokant ansah. Eine solche Verurteilung der Schauspiele führte konsequenterweise auch zur Verdammung ihrer „Prostitutierten“, ${ }^{30}$ die sich bei Ausübung ihres Metiers die Exkommunikation zuzogen. ${ }^{31}$

Ein weiterer Grund für die Ablehnung der Schauspiele dürfte in der Parodie christlicher Handlungen auf der Bühne bestanden haben. Einige Quellen berichten davon, dass vor allem das Taufritual und die Liturgie zum Gegenstand mimischer Nachahmung im Theater wurden. ${ }^{32}$ Ebenso karikierte man die Gottesmutter Maria, klerikale Personen oder Lehrstreitigkeiten unter den kirchlichen Autoritäten, ${ }^{33}$ so dass - wie es Gregor von Nazianz einmal übertrieben beklagte - „nichts in den Liedern oder Schauspielen populärer war, als der parodierte Christ““.34 Insbesondere die Erfahrungen aus der Zeit der Verfolgung, in der man sich bereits über die Christen mokiert hatte, mussten den Satiriker auf der Bühne in den späteren Jahrhunderten zum Objekt kirchlicher Kritik werden lassen. ${ }^{35}$

auch Jo. Mal. chron. 14.39(371). In Sev. Ant. hom. cat. 107 (= PO 25.4, 697) sind es sogar alle Sinne (Sehen, Hören, Schmecken, Tasten, Berühren), durch welche die Seele im Theater infiziert werde. Zur christlichen Kritik an heidnischer Instrumentalmusik siehe BEUCKMANN (1988) 64 Anm. 94 und zur Kritik am Tanz siehe ANDRESEN (1962).

27 Synes. calv. 9.3(72C); Chrys. hom. 1 in Jo. 2 (= PG 59, 26-27); Chrys. hom. 17 in Ephes. 3 (= PG 62, 119 -120); Aug. soliloq. 2.18; Marc. Diac. v. Porph. 38; Jac. Ser. hom. de spect. 2 (= Moss (1935) 103104); Bas. Sel. or. 27.2 (= PG 85, 313-316); vgl. Chor. Apol. mim. 10 -13, 26-27; WeBB (2008) 190 - 191. 28 WebB (2008) 215. Ihre Analyse basiert auf dem Artikel von LugARESI (2003b), der wiederum auf Goody (1997) zurückgreift.

29 Vgl. die Refutation dieses Klischees in Chor. Apol. mim. 26-27 u. 76.

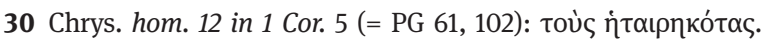

31 Cypr. epist. 2; Aug. fid. et op. 18.33 (= CSEL 41, 78); Conc. Elib a. $306 \S 62$ (ed. Suberbiola Martínez 1987); Conc. Arelat. a. $314 \S 4-5$ (= CC 148, 5); Const. apost. 8.32.9, 8.47.18 (ed. Metzger 1985, SC 320); Conc. Arelat. a. 442-502 § 20 (= CC 148, 118); vgl. auch Test. Dom. 2.2. (ed. lat. Rahmani 1899); Canon. Hipp. § 12 (= PO 31.2, 365); Hier. vita Hilar. 9.4-5; Pall. h. Laus. 37.

32 Aug. bapt. 7.53 .101 (= CSEL 51, 372-373); Chrys. hom. 6 in Mt. 7 (= PG 57, 71); LINK (1904) 10 - 16; vgl. auch WEISMANN (1975) 50.

33 Eus. v. C. 2.61.5; Socr. h.e. 1.6.35; Jo. Mosch. prat. 47; vgl. Cod. Iust. 1.4.4; Nov. 123.44. Auch Cyr. S. v. Euthym. 12.10 impliziert eine Kirchensatire auf der Bühne.

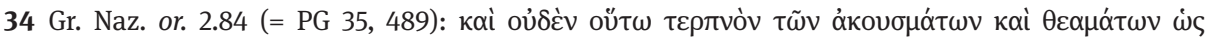

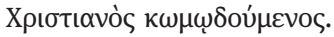

35 LEYerle (2001) 25-26. Aug. serm. 51.2 (= PL 38, 333) sieht einen Zusammenhang zwischen Martyrium und Bühnenparodie, indem der Märtyrer spectabatur illudendus. 
Die Parodie des Taufsakraments wie auch die Kritik an der Immoralität der Bühne scheinen in der Folge zur Herausbildung einer speziellen Form der Gegenpropaganda geführt zu haben, nämlich der Abfassung sogenannter „Taufmimus“-Geschichten und Erzählungen von „Märtyrerschauspielern“. In diesen Legenden, die gleichsam einen Gegenentwurf zum lästerlichen Schauspieler darstellen sollten, geht es stets um die wundersame Konversion von Bühnenakteuren: Entweder erfahren sie bei einer parodistischen Taufe auf der Bühne eine wahre Bekehrung, bekennen sich zum christlichen Glauben und werden daraufhin zu Märtyrern; ${ }^{36}$ oder die Umkehr erfolgt im Anschluss an ihre Theaterdarbietung durch andere Bekehrungserlebnisse und der Schauspieler wandelt sich durch Reue von einem personifizierten Symbol der Sünde zu einem wahren Streiter Christi. ${ }^{37}$ Vor allem Richard Lim hat in einer Analyse dieser Taufgeschichten von Schauspielern aufgezeigt, dass sie zwar inhaltlich auf die Zeit der Christenverfolgung zurückverweisen, jedoch zum Zeitpunkt ihrer Abfassung zwischen dem 4. und 6. Jahrhundert einen alternativen Gegensatz zwischen Theater und Christentum schaffen sollten, um besonders Katechumenen von den sündigen Schauspielen fernzuhalten. ${ }^{38}$

\section{Finanzielle Konkurrenz}

Ein weiterer Konflikt existierte auf der ökonomischen Ebene und findet in der kirchlichen Ermahnung zu Werken christlicher caritas ihren Ausdruck, d.h. der konkrete Wettstreit um private Gelder für kirchliche oder weltliche Projekte. ${ }^{39}$ Diese Kritik richtete sich vornehmlich an wohlhabende Eliten, die ihre euergetischen Tätigkeiten lieber christlichen Maximen wie der Spendung von Almosen widmen sollten als der Stiftung kostenreicher Spielveranstaltungen, seien es Theateraufführungen, Wagenrennen oder Arenawettkämpfe. ${ }^{40}$ Mit der Ermahnung zu Werken „,vortrefflicher Freigiebigkeit “41 ${ }^{\text {w1 }}$ wrde erneut eine diskursive Strategie eingesetzt, die auf einer moralischen Alternative basierte: Ausgaben für Spiele würden letztlich nur dem eigenen

36 Link (1904); VAn DER Vorst (1910); Weismann (1975); Cicu (2012) 178-179; Chron. Pasch. p. 513; Jo. Mal. chron. 12.49(314-315); Zu einem Bekehrungserlebnis während der Parodie eines Märtyrers siehe Men. Graec. III april. 17 (= PG 117, 408).

37 Anm. 402 Kapitel VII; Jo. Mosch. prat. 32. Eine weitere Variante besteht in der Tarnung gläubiger Menschen als Schauspieler, siehe Jo. Eph. vit. sanct. or. 52 (= PO 19.2, 166-178); Leont. N. v. Sym. $154-$ 155 (ed. Festugière 1974); Pall. h. Laus. 37. Vgl. auch Lim (2003) 91-97.

38 Lim (2003) 98-100; Weвв (2008)126-127. Vgl. Aug. serm. Den. 17.8 (ed. Morin, Misc. Agost. 1930 I); Stat. eccl. ant. § 24(86) (= CC 148, 171); Cyr. H. catech. 19.6.

39 French (1985) 96-101; Hugoniot (1996) 837-838; JimÉnEZ SÁnChez (2010) 263-267.

40 Ambr. off. 2.21.109 (= PL 16, 140); Paul. Nol. epist. 13; Aug. enarr. psalm. 147.7-8 (= CC 40, 21442145); 149.10 (= CC 40, 2184); Ast. Am. hom. 4.5-8 (ed. Datema 1970); Chrys. hom. 42 in Jo. 4 (= PG 59, 243-244); Bas. hom. Luc. 4.3 (= PG 31, 265-268); Sev. Ant. hom. cat. 26 (= PO 36.4, 549-550). Dieses Motiv ist noch bei Alcuin epist. 22 am Ende des 8. Jdts. zu finden.

41 Vgl. Ambr. off. 2.21.110 (= PL 16, 141): pulchra liberalitas. 
weltlichen Ruhm dienen und damit ephemeren Dingen gelten. Durch eine Verwendung der eigenen Mittel für christliche Werke der Nächstenliebe trüge man am Ende jedoch einen viel größeren, nämlich himmlischen Lohn davon. ${ }^{42}$

\section{Gegensätzliche Lebenswelten}

Die Konkurrenz zwischen weltlichem Festkalender und christlichen Feiertagen wird im folgenden Kapitel über die herrschaftliche Politik noch näher diskutiert, doch sie nimmt in vielen Texten der Kirchenväter eine zentrale Rolle ein. Letztlich gipfelte die zuvor beschriebene Kritik in einem Diskurs, der einen christlichen Lebenswandel ganz allgemein als unvereinbar mit dem Besuch oder der Förderung von Spielen konstruierte: „Entweder die Kanzel oder das Schauspielhaus“, wie es Jacob von Serugh einmal um das Jahr 520 auf den Punkt brachte. ${ }^{43}$ Um eine Identität christlicher Feste auszubilden, die ein Abbild der himmlischen Feier sein sollten, wurden die bisherigen Formen griechischer Feste als Gegenmodell benutzt. ${ }^{44}$ Der deutlichste Ausdruck dieser Dichotomie fand sich in der zeitgleichen Konkurrenz von Spielveranstaltungen und kirchlichem Gottesdienst wieder, die - wenn man den Zeugnissen verschiedener Kirchenväter Glauben schenken mag - an zahlreichen Orten auftrat. Salvian formulierte es folgendermaßen: spernitur dei templum, ut curratur ad theatrum; ecclesia vacuatur, circus impletur. ${ }^{45}$ Konkret ging es hierbei um die Anzahl der Teilnehmer an einer der beiden Lebenswelten: Gläubige kamen entweder gar nicht in die Kirche, weil eine Spielveranstaltung angesetzt war, oder sie verließen den Gottesdienst früher, um rechtzeitig am Spielort zu sein. ${ }^{46}$ In den Augen der kirchlichen Autoritäten wurde somit der Unterhaltung Priorität über den Kult und die christliche Lehrunterweisung eingeräumt, ${ }^{47}$ was einen ständigen Konflikt hervorrufen musste.

Im Übrigen fand sich eine derartige Auseinandersetzung nicht nur bei christlichen Klerikern, sondern spätantike Quellen des Judentums bezeugen ähnliche Vorbehalte. ${ }^{48}$ Im Talmud sind Verbote der Teilnahme an Wagenrennen, Schauspielen oder

42 Beispielhaft Chrys. de in. glor. 6-12 (ed. Malingrey 1972, SC 188) und Aug. c. acad. 1.2 (= CC 29, 4). 43 Jac. Ser. hom. de spect. 4 (= Moss (1935) 107); ähnlich auch Const. apost. 2.62 .1 (ed. Metzger 1985, SC 320) und die Ideenwelt von Johannes Chrysostomus, siehe LUGARESI (2008) 777-783. Diese

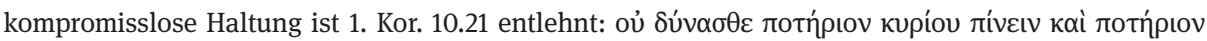

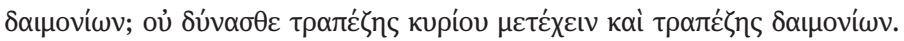

44 HARL (1981) 123-130.

45 Salv. gub. 6.7(38).

46 Vgl. Chrys. Anna 41 (= PG 54, 660); Chrys. theatr. 1-2 (= PG 56, 263-267); Leo M. serm. 71.1(84) (ed. Dolle 1973, SC 200); Aug. enarr. psalm. 80.2 (= PL 37, 1035); Reg. eccl. Carthag. 61 (= CC 149, 197); Caes. Arel. serm. 134.1. Zu dieser Konkurrenz um den favor populi siehe Lim (1999b).

47 Vgl. Chrys. Anna 41 (= PG 54, 660 -661); Chrys. Laz. 71 (= PG 48, 1043-1046); Chrys. hom. 7 in Mt. 5 (= PG 57, 78-79); Jo. D. parall. tit. 31 (= PG 96. 309-312); Salv. gub. 6.7(36).

48 JaCobs (1998); WeISS (2010); HÜBNER (1992) 112-113; GREATREX-WATt (1999) 13. 
Tierhetzen überliefert, die sich auf religiöse und moralische Argumente stützten. ${ }^{49}$ So war zum Beispiel die Teilnahme an Gladiatorenkämpfen aufgrund ihres Blutvergießens verboten und die Mitfeier heidnischer Feste bedeutete den Vollzug eines Götzendienstes. Die Ablehnung komischen Theaters gründete bereits auf den Worten aus dem ersten Psalm (1.1): „Wohl dem, der nicht dem Rat der Frevler folgt, nicht auf dem Weg der Sünder geht, nicht im Kreis der Spötter sitzt.“ Aber auch diese Kritik entstammte letztlich einem Diskurs religiöser Autoritäten, wohingegen in der Praxis viele Juden ohne Zweifel an traditionellen Spielen und Festen weiter teilnahmen. ${ }^{50}$

Sollte angesichts dieser Kritik also von einer grundsätzlichen Unvereinbarkeit zwischen kirchlicher Position und traditionellen Spielen, quasi einem unauflösbaren Konflikt, ausgegangen werden? Und inwiefern waren einige der soeben vorgebrachten Argumente überhaupt spezifisch für die Kritik am Spielewesen und beruhten nicht vielmehr auf allgemeinen Vorstellungen?

\section{Ambivalenzen und diskursive Brüche}

Als Antwort auf diese Fragen müssen zum einen die inhärenten Ambivalenzen und Brüche in dem so scheinbar festen, gegen die Spiele gerichteten Diskurs genauer herausgearbeitet werden - ein Umstand, der in der Forschung bislang wenig Beachtung gefunden hat. ${ }^{51}$ Es sind gerade die Überlagerungen mit der Welt des Spielewesens und ein ambivalentes Verhalten des eigenen Klerus, die in den Augen des zeitgenössischen Betrachters die Wirksamkeit der offiziellen kirchlichen Position relativiert haben dürften. Zum anderen geht es um generelle Spannungen, die sich aus dem Verhältnis zwischen christlichen Forderungen und dem spätantiken Alltagsleben ergaben und die entweder nur durch Missachtung oder pragmatische Kompromisse zu lösen waren. Im Zusammenhang mit der übergeordneten Fragestellung nach der Rolle von Spielen als Teil spätantiker Lebenswelten richtet sich somit das Interesse besonders darauf, wo sich die beiden Bereiche „Kirche“ und „Spiele“ überschnitten und inwiefern die im literarischen Diskurs hervorgebrachte Dichotomie in der alltäglichen Lebenspraxis aufgehoben wurde.

49 Honigman (2000); Jastrow (1950) 1663. Zur Datierung der talmudischen Texte zwischen dem 3. und 5. Jdt. siehe Stemberger (2010) 131; Stern (2010) 144-147.

50 Vgl. WeIss (1996) 444-446; WEISS (2010) 635-636; HEZSER (2010) 302-308. Auch Sitzinschriften bezeugen die Anwesenheit von Juden im Theater: IK 6.2 (Milet) 940.iii.g; IAph2007 2.18. Zur Mitgliedschaft in Zirkusparteien siehe Doctr. Jac. 1.40 (ed. DAGRoN-DÉroche (1991)); Jo. Mal. chron. 15.15 (389), 18.35(446); ReY-CoquaIs (1979) 167.

51 Die meisten der in Anm. 7 Kapitel II aufgeführten Studien beschäftigen sich mit der Darstellung von Konfliktpunkten, jedoch nicht mit den Gründen für das offensichtliche Scheitern des kirchlichen Vorgehens über einen Zeitraum von mehr als drei Jahrhunderten. 


\section{Übernahme bestehender Positionen}

Eine erste Feststellung besteht darin, dass die von den Kirchenvätern vorgebrachten Argumente nicht vollkommen neu waren, sondern entweder früherer Kritik antiker Bildungseliten entsprachen oder auf der Vorlage Tertullians beruhten, ${ }^{52}$ der allerdings mehr als ein Jahrhundert vor der Spätantike gelebt hatte. Diese Erkenntnis mag zunächst trivial erscheinen, dennoch dürften die religiös motivierten Argumente aufgrund der sich verändernden Kultpraxis zunehmend antiquiert und realitätsfern erschienen sein. Mit der fortschreitenden Christianisierung des Römischen Reichs wurden auch öffentliche heidnische Rituale immer weniger vollzogen. Auf der anderen Seite lässt das wiederholte Vorbringen moralischer Topoi diese zwar nicht obsolet werden, jedoch führt ein bereits in der Vergangenheit erfolgtes Scheitern schwerlich zu einem größeren Erfolg in der Gegenwart. Stereotype Vorstellungen dürften daher kaum eine große Überzeugungskraft besessen haben.

So lässt sich die Kritik an einer unkontrollierten Begeisterung für Wagenrennen oder an der moralischen Niedrigkeit von Schauspielen und ihrer Akteure bereits bei Autoren der späten Republik und der Kaiserzeit wiederfinden. ${ }^{53}$ Ebenso ist - wie im Fall des Pantomimus - die „Verweichlichung“ von Akteur und Publikum durch Schauspiel und Musik ein Topos, der sich von der griechischen Antike bis in das Mittelalter verfolgen lässt. ${ }^{54}$ Schon klassische Philosophen forderten eine verstärkte Konzentration auf die moralischen Aspekte des Lebens und eine Abkehr von ephemeren Handlungen wie Schauspielen oder niederen Vergnügungen, bei denen sie der mimesis zum Teil eine gefährliche Eigenschaft zuschrieben. ${ }^{55}$ Die Ähnlichkeit zum kirchlichen Diskurs der Spätantike ist unverkennbar, in dem sich antike und durch das Christentum beeinflusste Ideen trafen. Dementsprechend finden sich vergleichbare Positionen auch bei einigen zeitgenössischen paganen Autoren. ${ }^{56}$ Zum Beispiel stellte sich die Kritik von Libanius und Severus von Antiochia an der Amoralität der Wett-

52 Bereits die kurz nach Tertullian schreibenden Autoren Laktanz, Cyprian und Novatian nehmen ihn als Vorbild, vgl. LuGARESI (2008) 427-462.

53 Cic. rep. 4.20a; Sen. suas. 2.19; Plin. epist. 9.6; Tac. ann. 1.72; Val. Max. 2.6.7; D. Chr. 32; Hist. Aug. Heliog. 25.4; Lib. Or. 64.31 über Aelius Aristides. Dazu Cameron (1976) 223; SAllmann (1990) 246; EDWARdS (1997) 79-82; GARCÍA Moreno (2001) 10; Hugoniot (2004); LugARESI (2008) 172-178.

54 Pl. R. 3.398e-399a, 10.605e; Plut. Mor. 7.8.4(712E); D. Chr. 32.62; Clem. strom. 6.90.1-2 (ed. Descourtieux 1999, SC 446); Jul. Mis. 346 A u. 351D; Lib. Or. 64.38 u. 64.64; Cod. Theod. 2.8.23; Procop. Gaz. Pan. 16; Conc. Paris. a. 829 § 38 (= MANSI 14, 562); Dazu auch Theocharidis (1940) 34-37; Molloy (1996) 50 - 51; EdWARds (1997) 80; GUNDERSON (2000) 133; LEYERLE (2001) 19-20; PUCHNER (2002) 313; NAEREBOUT (2009).

55 Pl. R. 10.605c-606d; Epict. Ench. 33.2; M. Ant. 10.9; D. Chr. 32.20; Philostr. VS 1.25.9, 32.45; Porph. Abst. 19.4.5; WiEDEMANN (1995) 152-153; EdWARdS (1997) 84; Goody (1997) 103-104; OEPEN (2003) 203. Ebenso noch in einem fiktiven Dialog von Philosophen aus dem 6. Jdt. bei Aen. Gaz. Thphr. (= PG 85, 876 = ed. Colonna 1958, p. 3).

56 Amm. 14.6.18-20, 28.4.31-32; Jul. Ep. 84a(430B), Ep. 89b(304B-D); Claud. 19.338-364 (carm. in Eutr. II); Lib. Or. 3.11-12, Or. 19.28-29, Or. 35.13-14, Or. 42.8, Or. 45.20 -21; Zos. 1.6.1, 4.33.4. Ebenso Auson. lud. septem sap. $23-24$ (ed. Prete 1978, p. 139). 
kämpfe in Daphne fast identisch dar, obwohl Libanius von einem paganen Standpunkt aus die Sakralität der antiochenischen Agone erhalten wollte, während Severus als Bischof 130 Jahre später mit den gleichen Argumenten für ihre Abschaffung eintrat. ${ }^{57}$ Allerdings war schon die kaiserzeitliche Kritik an den Spielen bekanntermaßen selten auf fruchtbaren Boden gefallen, denn derartige Ansichten wurden vorrangig in Kreisen intellektueller Eliten gepflegt. ${ }^{58}$ Da die spätantiken Bischöfe in der Mehrzahl ebenfalls einem aristokratischen oder rhetorischen Milieu entstammten, ist davon auszugehen, dass ihre Argumente einen ähnlichen Eindruck erweckt haben. Sie dürften vor allem als eine Kritik rezipiert worden sein, die von Theologen geformt war und aus einer oberen Schicht heraus vorgetragen wurde.

Ein weiteres Stereotyp, das sich hinter dem Diskurs gegen das Spielewesen verbirgt, ist die durch biblische Texte und asketische Ideen begründete Vorstellung von der Frau als Verführerin zur Sünde, deren Sexualität es zu kontrollieren galt. ${ }^{59}$ Dieses Bild tritt besonders in der Kritik an den Bühnenschauspielen zutage, in der Tänzerinnen und Mimenschauspielerinnen in die Nähe von Prostituierten gerückt oder ganz explizit mit dem Ausdruck ró $\rho v \eta$ belegt wurden. ${ }^{60}$ Auch die Argumentation gegenüber dem Zuschauer stützte sich auf die Annahme, dass durch den Anblick von sich entblößenden Frauen eine Lüsternheit oder eine Abwertung der eigenen Ehefrau entstehen könne. ${ }^{61}$ Da diese Effekte auf einer von der Bühne ausgehenden Versuchung basierten, beschreiben manche Texte nicht nur die passive Seite des Zuschauers, sondern betonen auch eine aktive Rolle der Schauspielerin als Gehilfin des Verführers. ${ }^{62}$ Ein solches Bild beruhte aber vor allem auf einem biblischen Klischee, das seit Eva und Salome den Untergang des starken Geschlechts durch die Verführung des schwachen propagiert hatte. ${ }^{63}$ Diese Kritik lag also nicht in erster Linie in den Darbietungen selbst begründet, sondern gehörte zur traditionellen Vorstellung von der Frau als Verkörperung der Sünde, deren öffentliche Präsenz abgelehnt wurde. ${ }^{64}$

$\mathrm{Zu}$ Beginn dieses Kapitels wurde bereits auf die Abhängigkeit späterer Autoren von Tertullian und seiner Schrift de spectaculis hingewiesen. ${ }^{65}$ Auch hier stellt sich das Problem der Aktualität, denn schon in Tertullians Werk wirken die vorgebrachten Argumente hinsichtlich eines kultischen Charakters der römischen Spiele nicht mehr zeitgemäß, sondern akribisch konstruiert. Tertullian versuchte zu Beginn des 3. Jahrhunderts, durch detaillierte mythische Zusammenhänge und antike Symbolik den

57 Lib. Or. 10; Or. 53; Sev. Ant. hom. cat. 91 und 95 (= PO 25.1, 26 und 94-95).

58 Für die Spätantike vgl. Jul. Mis. 351C-D; Procop. Gaz. Pan. 29.

59 BRown (1988) 99; SCHAdE (2009) 216. Für die Kaiserzeit siehe EdWARDS (1997) 68.

60 Siehe Anm. 120 Kapitel III; vgl. auch Procop. Arc. 9; Brown (1988) 320; FrenCH (1998).

61 Zum Einfluss des christlichen Modells der ehelichen Keuschheit siehe Brown (1988) 308-309.

62 WEBB (2008) 170-171.

63 Webb (1997) 140; Hartney (2004) 57-58. Vgl. Soz. h. e. 20.1-2 zur Predigt von Johannes Chrysostomus über Herodias.

64 Weвв (2002) 299; WEBB (2008) 214-215 hat gezeigt, dass ähnliche Einstellungen zu Schauspielerinnen auch im heutigen Ägypten existieren.

65 LUGARESI (2008) 377-462. 
Ursprung der Spiele im heidnischen Kult nachzuweisen. Jedoch lässt gerade jene Detailliertheit die Unwissenheit seiner Leser vermuten und vermittelt den Eindruck einer antiquierten Argumentation, die höchstens noch gebildeteren Schichten bewusst war. ${ }^{66}$ So kommen als mögliche Quellen Tertullians bezeichnenderweise Schriften von Sueton und Varro in Betracht, die gleichfalls einen sehr kulturhistorischen Charakter aufgewiesen haben. ${ }^{67}$ Es ist daher davon auszugehen, dass Tertullians Argumente zwar einer tradierten Auffassung von der Geschichte römischer Spiele entsprachen, diese historischen Zusammenhänge und die genaue Symbolik den damaligen Zuschauern allerdings kaum mehr präsent waren. ${ }^{68}$ Tertullian selbst gesteht $\mathrm{zu}$ Beginn seiner Schrift ein, dass er gegen Unwissenheit (ignorantia) argumentiere und eine pagane Person die Spiele zunächst als eine reine Erfreuung (oblectatio) ansehen würde. ${ }^{69}$ Spiele wurden aufgrund von Prozessionen und Opferhandlungen zwar zum Teil noch als kultische Ereignisse rezipiert, darüber hinaus war es aber vor allem der soziale Anlass des Feierns und der Unterhaltung, welcher die Bewohner des Reiches in die Theater und Zirkusse strömen ließ. ${ }^{70}$ Umso weniger dürften also auch spätere Argumentationen, die sich an Tertullian orientierten, glaubwürdig erschienen sein. Am bezeichnendsten sind die Abschnitte über Wagenrennen, das Theater und das Amphitheater in den Etymologiae des Bischofs Isidor von Sevilla, auf die zu Beginn dieses Buches verwiesen wurde. ${ }^{71}$ Seine Schrift hatte zwar das primäre Ziel, ein Kompendium antiker Bildung und Lebenskultur zu überliefern, und wies somit per se einen antiquarischen Charakter auf, dennoch sind Isidors Ausführungen zu den verschiedenen Formen des Spielewesens nicht nur historisch, sondern auch von zeitgenössischer christlicher Kritik geprägt. ${ }^{72}$ Seine Argumente entnahm Isidor augenscheinlich der vierhundert Jahre zuvor verfassten Schrift Tertullians, was unter anderem an der Diskussion der nicht mehr existenten Gladiatorenspiele und an seiner Herleitung mythischer Ursprünge deutlich wird. Hier tritt offensichtlich zutage, dass nur noch ein literarischer Diskurs übernommen wurde, der keine Bezüge mehr zur Realität aufwies. $^{73}$

66 LiM (2009) 504-505.

67 Ville (1960) 288-289; CAStorina (1961) lxxxii-xci; CAMERon (1976) 58-59; Lim (2012) 68-69. 68 Vergleiche mit der Festkultur heutiger Tage liegen auf der Hand: Warum wurde z. B. der 1. Mai als Feiertag eingeführt, wann und von wem? Dieses Beispiel zeigt, wie hervorgehobene gesellschaftliche Anlässe zwar weiterhin eine soziale Identität formen bzw. gesellschaftliches Leben strukturieren können, sich die genauen Ursprünge aber leicht aus dem kollektiven Gedächtnis verlieren.

69 Tert. spect. 1; ebenso spect. 5: ignotis penes plures nostrorum.

70 Vgl. Kapitel III „Entsakralisierung der Spiele“.

71 Isid. Etym. 18.27-59.

72 Vgl. Mercado HeRnándeZ-SÁnchez Medina (2001) 221-222.

73 Dennoch haben seine Aussagen u.a. LiebeschueTz (2001b) 91 dazu verleitet, eine Existenz von allen Unterhaltungsgenres noch in Hispanien des 6. und 7. Jdts. anzunehmen, obwohl hierfür keine Zeugnisse vorliegen. Ähnliche Topoi finden sich in der Spätzeit auch bei Cassiod. var. 5.42.2-3. 


\section{Eine Abkehr der Spiele vom Heidentum}

Zugleich können aber eine Abkehr vom tertullianischen Diskurs und eine allmähliche Veränderung der christlichen Argumentation konstatiert werden. Es fällt auf, dass sich die Kirchenväter ab der Mitte des 4. Jahrhunderts immer mehr von einer religiösen Argumentation im Sinne Tertullians entfernten und stattdessen die moralische Unsittlichkeit der Spiele in den Vordergrund stellten. ${ }^{74}$ Ein frühes Beispiel findet sich in den Schriften des Firmicus Maternus um das Jahr 346: $:^{75}$ Firmicus spricht zwar vom Theater als Ort, wo die secreta istarum religionum zur Schau gestellt würden, und von heidnischen Priestern, die man zu Schauspielern mache. Anhand einer genauen Lektüre erkennt der Leser jedoch, dass es sich bei der Konfliktursache lediglich um die Darstellung von Mythen handelt und der „Tempel auf der Bühne“"76 in pathetischer Weise die Aufführung von Göttergeschichten umschreibt. Mit dieser Rhetorik beabsichtigte Firmicus zwar, einen Bezug zur paganen Religion herzustellen, aber dies konnte anscheinend nur noch auf der Ebene des Inhalts szenischer Aufführungen erfolgen, nicht mehr hinsichtlich einer realen Kultpraxis. Auch Johannes Chrysostomus belegt in zahlreichen Predigten die Schauspiele mit dem Adjektiv „satanisch“, tut dies aber aufgrund eines allgemeinen Zusammenhangs zwischen Sünde und Teufel, nicht in der Annahme einer kultischen Götterverehrung. ${ }^{77}$ So muss Augustinus an einer Stelle ganz offen zugeben, dass die Geschichten des Theaters über die Götter letztlich Fiktion seien: Das Theater sei verlogen, da es nichts Wirkliches hervorbringe und durch erdichtete Schandtaten sogar den Respekt vor den (heidnischen) Göttern missen lasse. ${ }^{78}$

Der Schwerpunkt verlagerte sich demnach auf eine Strategie, bei welcher anhand der Unmoral der dargestellten Mythen gegen die Lüsternheit des Theaters argumentiert wurde. ${ }^{79}$ In diesem Wandel der Kritik ist eine Reaktion auf die zur gleichen Zeit stattfindende Entsakralisierung der Spiele zu erkennen, die im nachfolgenden Kapitel

74 So schon Weismann (1972) 199-203; HaRL (1981) 130; und DeVoe (2003) 110 -111. Eine ähnliche Tendenz findet sich im rabbinischen Diskurs, dessen Autoritäten ebenfalls auf veränderte gesellschaftliche Rahmenbedingungen zu reagieren versuchten; dazu WEISs (1996) 452; WEISS (2010) 636.

75 Firm. err. 12.7-9.

76 Firm. err. 12.9: ad theatrum potius templum transferte.

77 Deutlich wird dies u.a. in Chrys. Laz. 7.2 (= PG 48, 1047), wo der Spielbesuch aufgrund von Tollheit, Lust, Geldgier und Liebe zu weltlichen Dingen verurteilt wird - jedoch eben nicht aufgrund von Götzendienst. Vgl. Analyse von NATALI (1975) 47-48.

78 Aug. civ. 2.10 (= CSEL 40, 72): Nam quod adfertur pro defensione, non illa [=scaenica] vera in deos dici, sed falsa adque conficta, id ipsum est scelestius si pietatem consulas religionis. Eine ähnliche Einschätzung findet sich in der semiotischen Abhandlung De Doctrina Christiana, in der Augustinus pantomimische Darbietungen als menschliche Einrichtungen bewusst von göttlichen Stiftungen unterscheidet (Aug. doctr. christ. 2.25.38[2.97])

79 Cypr. ad Donat. 8; Aug. civ. 7.26; serm. symb. 2.2 .3 (= PL 40, 638); Chrys. de in. glor. 38 (ed. Malingrey 1972, SC 188); Chrys. hom. 5 in Tit. 4 (= PG 62, 693); Sev. Ant. hom. cat. 95 (= PO 25.1, 9495). 
über die kaiserliche Politik näher diskutiert werden wird. Das Argument einer heidnischen Kultausübung vorzubringen, also im Sinne einer wirklichen Idolatrie, hätte kaum noch Sinn gemacht angesichts einer Schar von Gläubigen, die es aus eigener Erfahrung besser wusste. ${ }^{80}$ Zwar berichten Autoren noch bis in das 6. Jahrhundert hinein von Prozessionen, Hymnen, der Anrufung der Nike als Symbol des Sieges oder dem Anlegen besonderer Gewänder, ${ }^{81}$ doch waren alle diese Bräuche inzwischen ihrer ehemals kultischen Bedeutung beraubt und wurden in Form von „spectacles laïcisés“82 als volkstümliche bzw. traditionelle Elemente einer profanen Festveranstaltung weitergeführt. ${ }^{83}$ Allein die Verehrung städtischer Genien durch Teilnehmer von Festen und Spielen konnte noch einen religiösen Konflikt darstellen, da sie als Überbleibsel polytheistischer Vorstellungen angesehen wurden. ${ }^{84}$ Doch wie die Berichte hierüber nahelegen, wurde auch ein Lob der Stadtgottheit von christlichen Besuchern nun mehr als patriotischer Akt denn als religiöse Handlung aufgefasst. ${ }^{85}$

Der geschilderte, argumentative „Zwiespalt“ zwischen einer Nachfolge des tertullianischen Diskurses und einer Anerkennung der realen Verhältnisse, d.h. einer Berücksichtigung der inzwischen in der Gesellschaft vorherrschenden Kultpraxis, wird deutlich am Beispiel einer Predigt des Bischofs Severus von Antiochia aus dem Jahr 514: ${ }^{86}$ In dieser auf Syrisch überlieferten Schrift übt Severus heftige Kritik am Besuch von Wagenrennen und spricht ausführlich von moralisch verwerflichen und dämonischen Machenschaften. Umso bemerkenswerter muss er in einem Abschnitt seiner Rede zugeben, dass beim Schauspiel des Pferderennens keine wahre Anbetung von Dämonen mehr stattfinde und die Veranstaltung mehr einer Vergnügung gleiche. Sein Vorhaben, die eigenen Gläubigen vom Zirkusbesuch abzuhalten, erscheint dermaßen aussichtslos, dass Severus im weiteren Verlauf der Predigt sogar das Schicksal der Rennpferde und die an ihnen verübte Grausamkeit als Argument heranzieht. Diese singuläre Ausflucht in den Tierschutz ${ }^{87}$ darf als ein verzweifelter Versuch gedeutet

80 Diese Veränderung spiegelt sich u.a. bei Cassiodor wider, dessen Kritik sich ausschließlich auf den moralischen Bereich beschränkt, vgl. Cassiod. var. 4.51.6-11; FAUvINET-RANSON (2008) 152-153.

81 Lib. Descr. 12.8 (Antiochia); Bas. Sel. or. 27.1 (= PG 85, 308-313) (Seleucia/Kilikien); Sev. Ant. hom. cat. 26 (= PO 36.4, 549), hom. cat. 95 (= PO 25.1, 94) (Antiochia); Jos. Styl. chron. 30 (Edessa), hierzu TROMBLEY-WATt (2000) intr. xvi-xvii, xxxix.

82 NATAli (1975) 48.

83 Ein wohl ursprünglich religiöses Fest wie der Maioumas in Syrien scheint bereits um die Mitte des 4. Jdts. keine Opferhandlungen mehr gekannt $\mathrm{zu}$ haben, siehe Jul. Mis. 362D; ala2004 IV.25; BELAYCHE (2004b). Zur Fortführung von Bräuchen an den Kalenden siehe MACMulLEN (1997) 37-38; Hen (1995) 164; Meslin (1970) 70 - 93; MÜLler (1909a). Die Feier der Lupercalien am Ende des 5. Jdts. in Rom ist ein weiteres Beispiel, dazu Gel. pap. ep. adv. Andr. (= PL 59, 110 -116). Vgl. auch MARKUS (1985) 90; Rochow (1978) 486-87; HALPORN (1976) 101; Anm. 14 Kapitel II.

84 Sev. Ant. hom. cat. 26 (= PO 36.4, 543 u. 547); zur Reaktion Augustins siehe MARKUS (1991) 257268; GRAF (2008) 16-18.

85 Aug. serm. 62.9-10 (= CC 41Aa, 304-306).

86 Sev. Ant. hom. cat. 26 (= PO 36.4, 549-550).

87 Zugegebenermaßen beruft sich Severus bei seiner Argumentation auf Stellen aus der Bibel, anhand derer die Behandlung von Rennpferden widernatürlich und gegen Gottes Barmherzigkeit sei, 
werden, den eigenen Diskurs um weitere Argumente zu ergänzen, da die bisherigen Ermahnungen hinsichtlich dämonischer Machenschaften nichts gefruchtet hatten. Das Eingeständnis der realen Situation hatte der Rhetorik des Bischofs zuvor ihr Fundament entzogen. In diesem Sinne dürften auch Predigten anderer Kirchenväter, die mit einer Dämonisierung der Spiele argumentierten, keine wirkliche Überzeugungskraft besessen haben, da sich die Realität seit den Zeiten Tertullians verändert hatte. ${ }^{88}$ Zum einen waren Christen nun in der Mehrheit und mussten nicht mehr wie zuvor ihre eigene Identität von einer dämonischen Umwelt abgrenzen. ${ }^{89}$ Zum anderen hatten die Spiele einen Prozess der „Entsakralisierung“ durchlaufen und waren unter den christlichen Herrschern zu einem areligiösen Bestandteil der Kultur im Reich geworden. ${ }^{90}$ Ehemals heidnische Göttergeschichten lieferten zwar weiterhin den Inhalt von szenischen Schauspielen, ${ }^{91}$ doch stellte dieser Umstand für den äußeren Betrachter nun ein kulturelles und kein religiöses Problem mehr dar, zumal antike Mythen im literarischen Genre selbst durch christliche Schriftsteller gerne verarbeitet und rezipiert wurden. ${ }^{92}$

Auch die Kritik an der heuchlerischen mimesis des Schauspielers wurde nicht konsequent durchgehalten. ${ }^{93}$ Fand dieses Argument vor allem in Bezug auf das komische Theater Anwendung, so warnte man im Fall des Pantomimus vor den Effekten zu guter mimesis, welche die alten Mythengeschichten derart manifest werden lasse, dass die Zuschauer geradezu verführt würden. ${ }^{94}$ Manche Kirchenväter hegten sogar Bewunderung für die Kunst der Pantomimen und relativierten insofern die von ihnen selbst vorgebrachte Kritik. ${ }^{95}$ Letztlich dürfte die kategorische Ablehnung einer thea-

vgl. Prov. 12.10 („Der Gerechte weiß, was sein Vieh braucht, doch das Herz der Frevler ist hart“); Prov. 21.31 („Das Ross wird gerüstet für den Tag der Schlacht, doch der Sieg steht beim Herrn“); Psalm 145.16 („Du öffnest deine Hand und sättigst alles, was lebt, nach deinem Gefallen“); Sir. 18.13 („Das Erbarmen des Menschen gilt nur seinem Nächsten, das Erbarmen des Herrn allem Geschöpf“). Jedoch ist ein solches Argument gegen das Zirkuswesen meines Wissens nach einzigartig und findet bei keinem anderen Autor Verwendung. Allein Cicero äußerte einmal Mitleid mit Tieren in der Arena, nachdem bei von Pompeius organisierten venationes die Tiere massenhaft abgeschlachtet wurden (Cic. fam. 7.1.3).

88 Vgl. CRAmer (1980) 105 in seiner Analyse der Predigten des Jacob von Serugh.

89 Siehe MARKuS (1993) 101.

90 Siehe hierzu Diskussion in Kapitel III „Entsakralisierung der Spiele“.

91 Aug. civ. 7.26; Bas. Sel. or. 27.1 (= PG 85, 312); Sev. Ant. hom. cat. 95 (= PO 25.1, 94-95).

92 JüRgens (1972) 32-35; LiEBESCHUETZ (1995); Liebeschuetz (1996b); LEYERLE (2001) 31; CAMERON (2007) 30. Beispiele hierfür sind Fulg. myth. (dazu Hays (2002); Ferguson (2006)); Aen. Gaz. Ep. 17 (ed. Massa 1962); Syn. provid. 13.9; Nonn. (dazu Lind (1935); CAMERon (1965)); Lyd. mens.; Chor. Apol. mim. 155-158; vgl. auch Index in GARZYA-RoQUES (2000) 448.

93 Zumal das Bild des Schauspielers als Lügner auf klassische Vorurteile zurückging, dazu EDWARDS (1997) 79.

94 Z. B. Jac. Ser. hom. de spect. 5 (= Moss (1935) 108-112); Sev. Ant. hom. cat. 95 (= PO 25.1, 94-95); vgl. WEBB (2008) 183-194.

95 Aug. civ. 22.24.3; WeISMANn (1972) 110 - 11 u. 175-176; CrAmER (1980) 103; WeBB (2008) 190 - 196; VANDENBERGHE (1955) 35. 
tralischen mimesis kaum von dem überwiegenden Rest der Gesellschaft geteilt worden sein, für den das Theater und die Lektüre klassischer Dramen weiterhin zum Bestand traditioneller kultureller Aktivitäten gehörten. ${ }^{96}$

\section{Das asketische Bild der pompa diaboli}

Ein weiteres, dem kirchlichen Diskurs inhärentes Problem bestand in seiner Prägung durch asketische Ideale. In der Tat entstammten einige der patristischen Autoren einem Milieu von Asketen und hatten vor ihrem Bischofsamt ein Leben als Mönch geführt. ${ }^{97}$ In ihrer späteren Funktion als allgemeine Seelsorger gaben sie aber ihre asketischen Positionen nicht einfach auf. Johannes Chrysostomus mag hierfür als Paradigma dienen, indem er als erbitterter Gegner der Spiele nach seiner Berufung zum Bischof in Antiochia und Konstantinopel eine dem Mönchstum nachempfundene Bekehrung der urbanen Lebensführung forderte. ${ }^{98}$ Bezeichnenderweise erschienen dem Asketen Hilarion in der syrischen Wüste die Angriffe der Dämonen in Form von städtischen Rennpferden und Gladiatoren. ${ }^{99}$ Weitere Texte gegen das Spielewesen hoben ebenso den Gegensatz zwischen der sündigen Stadt mit ihren Schauspielen und dem reinen Leben außerhalb davon hervor. ${ }^{100}$ Gerade in den Schriften des Chrysostomus wurde ein vollständig alternatives Gesellschaftsbild konstruiert, dem das herkömmliche kulturelle Leben diametral entgegenstand. ${ }^{101}$ Manche Forderungen an die Zuhörerschaft waren somit von einer radikalen Vorstellung geprägt, die ein normaler Gläubiger in dieser Form schwer teilen oder erfüllen konnte. ${ }^{102}$ Wohl deshalb

96 Vgl. die Argumentation bei Chor. Apol. mim. 10-13 u. 83-91; dazu MalineaU (2005) 167. Auch Augustinus sah Dramen als festen Bestandteil des Unterrichts an (civ. 2.8). Ebenso demonstriert ein Epigramm aus dem 6. Jdt. noch die Popularität herkömmlicher mimesis (AP 6.56). Zur kulturellen Verortung des Theaters siehe Kapitel VII „Die kulturelle Verortung szenischer Darbietungen“.

97 Siehe hierzu die neueste Studie durch Liebeschuetz (2011b).

98 Chrys. hom. 68 in Mt. 3 - 4 (= PG 58, 643-646); Chrys. hom. 7 in Heb. 4 (= PG 63, 66 - 68); vgl. Zach. Schol. vit. Sev. 51-52 (ed. engl. Ambjörn 2008); vgl. FrenCH (1985) 96; BRown (1988) 315-316; SANDWELl (2004) 43 u. 51-52; LIEBESCHUETZ (2011b) 205-215.

99 Hier. vita Hilar. $3.8-12$.

100 Nil. exerc. 4 (= PG 79, 722-723); Chrys. pan. mart. 11 (= PG 50, 647); Hier. ep. 58.4; HARL (1981) 134-136; vgl. auch eine Zusammenstellung bei Jo. D. parall. tit. 31 (= PG 96. 307-312). Es ist aber bezeichnend, dass gerade prominente Autoren wie Augustinus, Johannes Chrysostomus oder Severus von Antiochia in ihrer Jugend selbst dem Besuch der Schauspiele zugetan waren, vgl. Aug. conf. 3.24; Chrys. sac. 1.4 (= PG 48, 624) mit VAndenBeRghe (1955) 35; Zach. Schol. vit. Sev. 51 (ed. engl. Ambjörn 2008). Zum konstruierten Gegensatz zwischen Asket und Stadtleben siehe SARADI (2006) $102-111$.

101 HARL (1981) 134-135; HARTNEy (2004) 48-51; LiebeschuetZ (2011a) 321-323; LiebesChuetZ (2011b) 185-204.

102 So entgegnet Johannes Chrysostomus auf den Wunsch von Gläubigen nach Belustigung ( $\delta$ เ $\alpha$ -

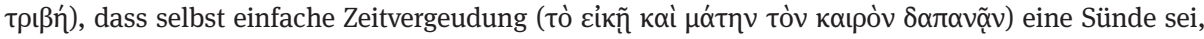
siehe Chrys. hom. 37 in Mt. 7 (= PG 57, 428); vgl. auch Chrys. hom. 15 in Heb. 4 (= PG 63, 121-124). 
warfen Gläubige Johannes Chrysostomus unter anderem vor, durch seine unablässige Kritik am Theater die ganze Weltordnung invertieren zu wollen. ${ }^{103}$ Sich außerhalb der urbanen Gemeinschaft zu stellen und zudem allen weltlichen Vergnügungen zu entsagen, war sicherlich der Anspruch an einen Mönch, jedoch nicht an einen gewöhnlichen Stadtbewohner. ${ }^{104}$

Eine ebenfalls der Askese entstammende, stereotype Vorstellung findet sich im Motiv der pompa diaboli wieder. Dieser Begriff hatte seinen Ursprung in erster Linie im Taufbekenntnis, wo die Bewerber der pompa des Teufels widersagen mussten. ${ }^{105}$ Obwohl dieser Terminus zunächst nur eine Prozession bezeichnete, erlangte er durch das christliche Taufritual die Semantik einer diabolischen Versuchung und wurde so im Laufe der Zeit zu einem Symbol für jegliches vom Teufel bestimmtes Verhalten seien es Wagenrennen, Schauspiele im Theater, weltliche Vergnügungen oder allgemein unsittliche Handlungen. ${ }^{106}$ Die Texte zeigen deutlich, wie dieser Begriff argumentativ generalisiert wurde, ohne in irgendeiner Weise eine theologische Differenzierung der einzelnen Arten von Versuchungen vorzunehmen: ${ }^{107}$ „Christians came to use it in the broader sense of anything that they saw as connected with the realm of the devil [...] Thus we can see Chrysostom defining the huge areas of life that should be seen in religious terms“. ${ }^{108}$ In diesem Sinne kann die pompa diaboli als ein „Schlagwort“ der spätantiken Kirche bezeichnet werden, das aufgrund seines undifferenzierten Charakters aber kaum pastorale Wirkung entfalten konnte. Wenn jegliche Spielgattung im wahrsten Sinne des Wortes verteufelt wurde, stellten sich dem Zuhörer am Ende nur zwei radikale Alternativen: Entweder der Sache des Teufels gänzlich zu entsagen oder den Begriff der pompa als ein reproduziertes Klischee zu ignorieren. So mag auch hier die Radikalität der Forderung an die Gläubigen selten zu der gewünschten Reaktion geführt haben.

103 Chrys. hom. 37 in Mt. 6 (= PG 57, 427).

104 LUGARESI (2007) 24: „Si ritorna così, in qualche modo, alla necessità di escluderli definitivamente dall'orizzonte civile.“ So auch NATALi (1975) 41 u. 59, der von einem Dualismus zwischen christlicher und stadtbürgerlicher Identität spricht. Ähnlich BROwN (1988) 337.

105 Quodv. symb. 1.1-2 (= CC 60, 305-310); Sev. Ant. hom. cat. 54 (= PO 4.1, 48-49); hom. cat. 95 (= PO 25.1, 94); ALÈs (1910); HARL (1981) 133.

106 Quodv. symb. 1.1-2 (= CC 60, 305-310); Salv. gub. 6.6(31); Caes. Arel. serm. 12.4; Conc. Arvern. a. 535 § 8 (= CC 148 A, 107); Chrys. hom. in I Cor. 7:2 2 (= PG 51, 210 -211); Chrys. David 32 (= PG 54, 696); Cyr. H. catech. 19. 6. Dazu vor allem Waszink (1947); Binder (1998); und KaHLos (2005).

107 Z. B. Chrys. cat. 5 (2) 5 (= PG 49, 239): Schauspiele, Zirkusspiele, alle Sünden, Augurenschau,

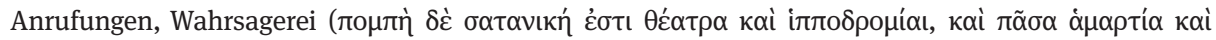

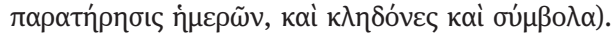

108 SANDWELL (2004) 51-52. Dies wurde sogar auf den Thermenbesuch ausgeweitet, vgl. VANDENBERGHE (1955) 38; MAGOULIAS (1971) 233-238. 


\section{Kaiserliches Handeln}

Eine weitere Schwäche der kirchlichen Argumentation bestand in der gänzlichen Abwesenheit des Kaisers in der patristischen Kritik. Obgleich unzählige Predigten und Traktate zu einer Abkehr von den Spielen aufriefen und Gläubigen den Besuch untersagten, ist es bemerkenswert, dass der wohl bedeutendste Besucher von Spielveranstaltungen keine explizite Erwähnung findet. Schließlich bestand ein weitverbreiteter Aufruf der Kirchenväter darin, die eigenen Gläubigen zu einem konsequenteren Zeugnis nach außen zu bewegen. ${ }^{109}$ Nicht nur aufgrund seines persönlichen Handelns, sondern auch in seiner Funktion als treibende finanzielle Kraft hätte man daher eine ähnliche Kritik an der Person des Kaisers erwartet. Allerdings verbleiben fast alle Texte auf der Ebene allgemeiner Ermahnungen oder richten sich konkret an die im Gottesdienst versammelte Gemeinde. Gelegentlich findet sich eine Kritik an Stadtbeamten oder Provinzstatthaltern, die Spielveranstaltungen unterstützten, doch erstaunlicherweise scheint keine einzige Quelle vorzuliegen, in der direkt Kritik am Kaiser geübt worden wäre. ${ }^{110}$ Offenbar wollte man den Vorwurf der Majestätsbeleidigung vermeiden, auf welchen konsequent vorgebrachte Forderungen hinausgelaufen wären. Außerdem waren sich wohl auch die Kirchenväter der Bedeutung von Spielen für den römischen Kaiser bewusst, wie sie in den folgenden Kapiteln noch weiter ausgeführt wird. ${ }^{111}$ Das persönliche Verhalten des Kaisers legitimierte wiederum die Handlungen seiner Untertanen vor Ort, so dass sich hinter dem Schweigen über die Figur des Herrschers auch eine bewusste Argumentationstaktik erkennen lässt, die darauf abzielte, nicht ein Argument gegen die eigene Sache vorwegzunehmen. In der Tat instrumentalisierte zum Beispiel der christliche Rhetor Choricius das Verhalten des Kaisers als ein Argument zur Verteidigung des Mimus, ${ }^{112}$ und auch die große Anzahl der Gläubigen dürfte in der Praxis rechtfertigend auf den Kaiser als gesellschaftliches

109 Chrys. Laz. 7.1 (= PG 48, 1043-1046); theatr. 1 (= PG 56, 263-265).

110 Die einzigen mir bekannten Beispiele stammen von Johannes Chrysostomus: Chrys. hom. 12 in 1

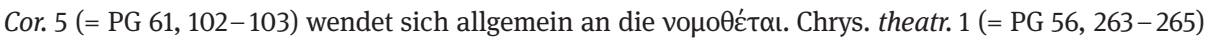
kritisiert die Teilnahme an Zirkusspielen auch von „denjenigen, die im oberen Teil sitzen“ ( $\tau \tilde{\omega} v \mu \varepsilon v$

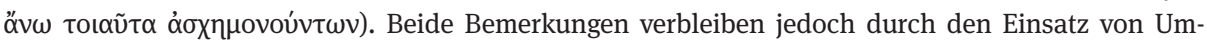
schreibungen auf der Ebene einer indirekten Kritik. Zumindest im Zuge einer Statuenaufstellung für die Kaiserin Eudoxia im Jahr 403/04, wo es zu einer Darbietung von Pantomimen und Mimen kam, reagierte Johannes anscheinend mit einem verurteilenden Predigtzyklus, dazu Soz. h. e. 8.20.1-2; SETTON (1941) 178-180. In seiner Schrift gegen Symmachus forderte Prudentius Kaiser Arcadius dazu auf, den Gladiatorenkämpfen ein Ende zu bereiten (Prud. c. Symm. 2.1091-1129), und Priscian lobte Kaiser Anastasius 100 Jahre später für das Verbot grausamer venationes (Prisc. Anast. 223-227). Allerdings wird auch bei diesen direkten Ansprachen keine grundsätzliche Kritik am persönlichen Verhalten des Kaisers geübt.

111 Vgl. Isid. Pel. epp. 5.185(1469) (ed. Évieux 2000, SC 454).

112 Chor. Apol. mim. 58-59. In Antiochia scheinen Gläubige auf den Stadtrat als Rechtfertigung verwiesen haben, vgl. Chrys. hom. 37 in Mt. 6 (= PG 57, 427). 
Vorbild verwiesen haben. ${ }^{113}$ So blieb die kirchliche Argumentation in dieser Hinsicht ebenfalls ihrer eigenen Ambivalenz verhaftet.

\section{Verhältnisse im Klerus}

Die Tatsache, die wohl am meisten den Diskurs gegen das Spielewesen unterminierte, war allerdings das Fehlverhalten des Klerus selbst. Auch wenn die zahlreichen Gegenreden der Kirchenväter den Eindruck einer geschlossenen Position zu vermitteln suchen, sind es gerade Zeugnisse aus anderen Textgattungen, die für den spätantiken Alltag eine weitreichende Teilnahme von Klerikern an den Spielen vermuten lassen; dies sowohl auf der niederen Ebene von Diakonen, Mönchen und Presbytern als auch im Fall von Bischöfen. In sämtlichen Regionen des Reichs zwischen dem 4. und dem 7. Jahrhundert - und sogar noch in frühmittelalterlicher Zeit - lassen sich Konzilsbeschlüsse finden, welche den Besuch von weltlichen spectacula oder von Schauspielen im privaten Rahmen durch Kleriker untersagen und ihn somit gleichzeitig bezeugen. ${ }^{114}$ Selbst in den eigenen Familien scheint der kirchliche Antidiskurs nicht den gewünschten Niederschlag gefunden zu haben, wie es ein entsprechendes Verbot für die Söhne von Klerikern bezeugt. ${ }^{115}$ Literarische Quellen berichten sogar von Priestern und Bischöfen, die privat mit Schauspielern, Pantomimen oder Wagenlenkern Umgang hätten ${ }^{116}$ - einer Personengruppe, die eigentlich außerhalb der kirchlichen Communio stand. ${ }^{117}$ Mögen manche dieser Argumente in innerkirchlichen Auseinandersetzungen gegen Gegner instrumentalisiert worden sein und daher nicht in jedem Fall der Realität entsprochen haben, ${ }^{118}$ scheint das Beispiel eines Bischofs,

113 Vgl. die Diskussion bei Epict. Fr. 3.4 über das Verhalten eines Statthalters im Theater. 114 Decret. Euseb. 4 (= MANSI 2, 426); Conc. Laod. a. $343-81 \S 54$ (= MANSI 2, 574 u. 582); Conc. Ven. a. 461-491 § 11 (= CC 148, 154); Conc. Agath. a. $506 \S 39$ (= CC 148, 209-210); Mart. Brac. canon. 60 (ed. Barlow 1950); Sisebut. epist. ad Euseb. (= MGH Epp. III p. 668-669); Conc. Trullo a. 692 § 24 (= PG 137, 592). Vgl. auch Jo. Gaz. 836 (ed. Renault 2002, SC 468). Zur Zeit Karls des Großen gleicht der Wortlaut immer noch dem der spätantiken Konzilien, siehe Conc. Mogunt. a. 813 § 10 (= MANSI 14, 67); Conc. Tur. a. $813 \S 7$ (= MANSI 14, 84); Conc. Aquisgr. a. $816 \S 83$ u. 100 (= MANSI 14, 202 u. 210); Conc. Paris. a. $829 \S 38$ (= MANSI 14, 562). Marc. com. chron. a. 526 (= MGH Auct. ant. XI p. 102) berichtet von Bischof Euphrasius, der bei einem Erdbeben in Antiochia vom Obelisken des Hippodroms erschlagen worden sei; er muss folglich anwesend gewesen sein.

115 Conc. Carth. a. $397 \S 15$ = Coll. Hisp. 11 (= CC 149, 332); Ruric. epist. 2.24- 43.

116 Const. apost. 8.47.18 = can. apost. 18 (ed. Metzger 1985, SC 320); Gr. Naz. ep. 193 (= PG 37, 316); Pall. v. Chrys. 15.66, 15.88-92, 16.53-58 (ed. Malingrey-Leclercq, SC 341); AOC III p. 96-97; Pall. $h$. Laus. 26.

117 Siehe Anm. 31 Kapitel II; LIM (2003) 87-88. In den Akten der Heiligen Xanthippa, Polyxena und Rebecca aus der Mitte des 3. Jdts. wird sogar eine direkte Identifikation von Dämonen mit Schauspielern vorgenommen, siehe Montague Rhodes (1893) 73 cap. 21.

118 In der Vita des Johannes Chrysostomus wird von seinem Gegenspieler Porphyrius berichtet, dass er als Priester in Antiochia Umgang mit Wagenlenkern und Pantomimen gehabt habe (Pall. $v$. Chrys. 16.53-58; ed. Malingrey-Leclercq, SC 341). Allerdings ist bereits die Anfangsbeschreibung des 
der sich mit dem Spielewesen assoziierte, nicht gänzlich unglaubwürdig gewesen zu sein. So musste im Jahr 534 sogar der Kaiser persönlich durch einen Erlass einschärfen, dass Diakone, Priester und Bischöfe nicht Schauspielen oder Wettrennen beiwohnen sollten. ${ }^{119}$ Dort hatten sie wohl, wie aus dem Text der Konstitution hervorgeht, als Parteianhänger derart mitgefiebert, dass sie sich an der Provokation anderer Zuschauer beteiligten. Es sind zudem Fälle überliefert, in denen Bischöfe durch eigenen Einfluss oder kirchliche Gelder zur Realisierung von Schauspielen beigetragen haben sollen ${ }^{120}$ oder in denen Kleriker selber Theater spielten. ${ }^{121}$ Diese Zeugnisse erlauben durchaus, die scheinbar feste und einheitliche Position der kirchlichen Autoritäten zu hinterfragen und einen Blick auf die tatsächliche Verhaltensweise des Klerus an vielen Orten zu werfen. In der Praxis dürften sich kirchliche Amtsträger wahrscheinlich oft an dem Verhalten der eigenen Gläubigen orientiert und in einer Teilnahme an Spielen keine verwerfliche Handlung gesehen haben. ${ }^{122}$

$\mathrm{Zu}$ dieser Annahme passt, das sich kirchliche Autoritäten im Alltag eher tolerant oder sogar unwissend gegenüber dem Problem der Spiele verhielten. Eine interessante Episode findet sich in der Vita des Mönchs Hypatios, die um die Mitte des 5. Jahrhunderts verfasst wurde: ${ }^{123}$ In ihr wird berichtet, dass der Stadtpräfekt von Konstantinopel, Leontius, um das Jahr 434 plante, in Chalcedon wieder einen Olympischen Wettkampf ('O $\lambda \dot{u} \mu \tau \iota \alpha)$ einzurichten. Als Hypatios davon erfuhr, wollte er den lokalen

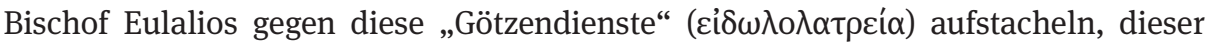
aber ließ zunächst nur eine verhaltene Reaktion erkennen. Hypatios mobilisierte daraufhin mehrere Geistliche, doch bevor es zur Konfrontation kam, zog der Präfekt sein Vorhaben zurück. Nun aber erst, so heißt es, wollte Hypatios genauer wissen, was die Olympischen Wettkämpfe ausmache und was an ihnen so schlecht sei, da er dies nur vom Hörensagen her kannte. ${ }^{124}$ So wurde er schließlich durch einen gewissen

Porphyrius bewusst negativ gehalten, da er als späterer Bischof die Anhänger von Johannes verfolgte. Auch die Rechtmäßigkeit seiner Bischofsweihe wird durch den Verfasser Palladius angezweifelt. In Apamea wiederum brachten lokale Kleriker ihren Bischof anscheinend mit Schauspielerinnen in Verbindung, weil er Monophysit war (AOC III p. 96-97). Siehe auch MAGoulias (1971) 249.

119 Cod. Iust. 1.4.34.

120 Vgl. die Eingabe an Papst Leo und an die Synode von Chalcedon über den Bischof von Alexandria (= MANSI 6, 1013). Patriarch Gregor von Antiochia soll sich bei Tiberius II. persönlich für den Neubau eines Zirkus eingesetzt und anschließend Mimen nach Antiochia gebracht haben, siehe Joh. Ephes. hist. eccl. 5.17 (ed. lat. Brooks 1936 = CSCO 3.3, 202-203)

121 Val. Abb. ordo quer. 34 (= PL 87, 444); Pall. v. Chrys. 15.88-92 (ed. Malingrey-Leclercq, SC 341). Vgl. auch Gr. Naz. ep. 246 (= Bas. ep. 169).

122 Gleiches kann bereits für die Kaiserzeit konstatiert werden, als Angehörige der Elite oder die Kaiser selbst zuweilen die infame Arena oder die Theaterbühne betraten, siehe EDwARDS (1997) 8590.

123 Call. v. Hyp. 33 (ed. Bartelink 1971, SC 177). Zu dieser Episode siehe auch JimÉnEZ SÁNCHEZ (2013).

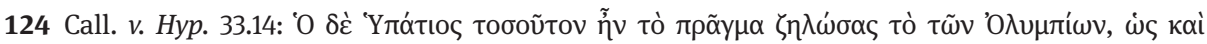

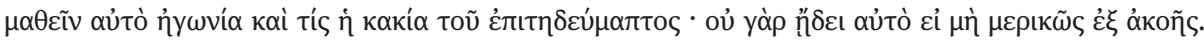


Eusebios in einem Brief belehrt, dass die Olympischen Spiele ein Fest Satans und einen für Christen verderblichen Götzendienst darstellten.

Entsprechend der Intention dieser Geschichte musste die Rolle des Hypatios als Kämpfer gegen das Böse hervorgehoben werden und es ist daher für den Leser nicht erstaunlich, dass erst seine Initiative Chalcedon vor dem Unglauben bewahrte. Die Charakterisierung des lokalen Bischofs als indifferent mag insofern der Absicht des Erzählers ein wenig angepasst worden sein. Aber vielleicht war eine solche Haltung auch nichts Ungewöhnliches, sondert schildert eher den Normalfall eines Seelsorgers, der in der Einrichtung von neuen Bühnenwettkämpfen kein größeres Problem sah. ${ }^{125}$ Gestützt wird diese Vermutung auf die nachträgliche und eigentlich überflüssige Schilderung von Hypatios' Unwissenheit. Obgleich ein leidenschaftlicher Kämpfer gegen das Theater, scheint er von den eigentlichen Inhalten und - was noch schwerer wiegt - von der eigentlichen Problematik in Wahrheit keine Ahnung gehabt zu haben, so dass in dieser Episode sein Kampf gegen die Spiele als eine fast ignorante Position erscheint. Offenbar nahmen einige Kleriker an den Spielen kaum Anstoß und zeigten sich in ihrer seelsorgerischen Praxis kompromissbereit: Spielveranstaltungen waren Bestandteil einer traditionellen antiken Stadtkultur und ein Ankämpfen dagegen wäre aussichtlos gewesen. Es galt, beide Lebensbereiche zwar gegeneinander abzugrenzen, jedoch auch Verständnis für das Verhalten vieler Gläubiger aufzubringen. Selbst Augustinus, dessen Schriften eine erhebliche Polemik gegen die Spiele aufweisen, lässt an einer Stelle einen solchen Kompromiss aufscheinen: Abstinete vos, quantum potestis (!), a nugatoriis spectaculis. ${ }^{126}$ Auch ihm muss bewusst gewesen sein, dass man tradierte Lebensgewohnheiten schwerlich ändern konnte und seine eigenen Appelle daher nur bedingte Wirkung entfalten würden. ${ }^{127}$ Die Veränderung antiker Lebensrhythmen und -gewohnheiten konnte nur im Rahmen eines allmählichen und langsamen Prozesses erfolgen. In der Tat findet sich im Kontrast zu den zahlreichen Ermahnungen der Kirchenväter kein Bericht, der in positiver Weise von einer erfolgreichen Konversion der Gläubigen und der Abschaffung einer Spielveranstaltung direkt berichten würde. ${ }^{128}$

125 Laut der Erzählung war die Ausrichtung der Spiele im Theater geplant, so dass es sich wohl um einen musischen Wettbewerb gehandelt hätte. JIMÉNEZ SÁNCHEZ (2013) 44 scheint hingegen eher einen athletischen Wettkampf zu vermuten.

126 Aug. serm. 9.17 (= CC 41, 141).

127 Zumal sich Augustinus in seiner Jugend selbst von den Emotionen im Theater mitreißen ließ, vgl. Aug. conf. 3.2-4. Allerdings hat MARKUS (1991) aufgezeigt, dass sich die Position Augustins nach religiösen Ausschreitungen im Jahr 399 verschärfte. Auch bei Philon und Clemens von Alexandrien, die in einer Stadt mit großer Begeisterung für das Theater lebten, lässt sich eine eher moderate Kritik konstatieren, siehe LUGARESI (2008) 463-481 u. 489-491.

128 Von Augustinus stammt das einzig bekannte Zeugnis, wenngleich von zweifelhafter Natur, denn ausgerechnet in seiner Bischofsstadt Hippo sollen die Schauspiele fast gänzlich verschwunden gewesen sein (pene defecerunt); laut seiner Predigt sei man auch in der Nachbarstadt Simitthu nicht mehr ins Theater gegangen (Aug. serm. Den. 17.7-9 = ed. Morin, Misc. Agost. 1930 I). Zwei Zeugnisse aus Gaza des 6. Jdts. legen nahe, dass die kirchliche Autorität dort gewisse Einschränkungen beim 


\section{Integration der Spielräume}

Ließen sich in der Praxis also viele Kleriker auf die Spiele ein oder förderten sie sogar, zeugen weitere Beispiele von der aktiven Instrumentalisierung der Spielräume für kirchliche Belange und von ihrer Durchdringung mit christlichen Verhaltensweisen. Obgleich offiziell tabu, wurden das Theater oder das Hippodrom in ihrer Funktion als öffentliche Räume gelegentlich auch durch Mitglieder der Kirche für politische Anliegen genutzt: ${ }^{129}$ Als die Partei der Nestorianer auf dem Konzil von Ephesos im Jahr 431 die Absetzung der orthodoxen Bischöfe anstrebte, hängte sie eine Bekanntmachung der angekündigten Absetzung im Theater auf, wovon man sich wohl ein Höchstmaß an Öffentlichkeit erwartete. ${ }^{130}$ Im Circus Maximus wiederum organisierten die Zirkusparteien im Jahr 357 mit Akklamationen eine Demonstration der Unterstützung für den zurückkehrenden Papst Liberius, nachdem Constantius II. dessen Amnestie verkündet hatte. ${ }^{131}$ Gegen die Ernennung eines arianischen Germanen zum Caesar durch Leo I. demonstrierten sowohl der Patriarch als auch Mönche im Hippodrom von Konstantinopel. ${ }^{132}$ Religiöse Konflikte konnten bei Bedarf also durchaus über die Foren des Spielbetriebs ausgetragen werden, indem man zum Beispiel die Anhänger der Zirkusparteien instrumentalisierte oder die Taktik von Theaterclaqueuren übernahm. ${ }^{133}$ Papst Damasus und Bischof Marcellus von Apamea scheinen im 4. Jahrhundert sogar auf Gladiatoren und Wagenlenker zurückgegriffen $\mathrm{zu}$ haben, um mit ihnen als Schlägertruppen eigene Forderungen durchzusetzen. ${ }^{134}$ Umgekehrt legen Graffitti aus der Nähe von Bostra und aus Priene nahe, dass die Rivalität der Zirkusparteien selbst im kirchlichen Raum präsent war und dort vom Klerus toleriert wurde. ${ }^{135}$ Eine kaiserliche Anordnung aus dem Jahr 459 offenbart schließlich, dass christliche Objekte

Besuch nächtlicher Schauspiele hatte durchsetzen können (Chor. Apol. mim. 103-109; Jo. Gaz. 836 = ed. Renault 2002, SC 468). Aber auch in diesem Fall ist von keiner Abschaffung die Rede, vielmehr beabsichtigte der Stadtpräfekt von Gaza eine Rückkehr zum alten Zustand.

129 Bischof Severus aus dem syrischen Gabala akzeptierte z. B. das Theater durchaus als einen öffentlichen Ort, wo Kaiserbilder aufzuhängen seien (Sever. creat. $6.5=$ PG 56, 489).

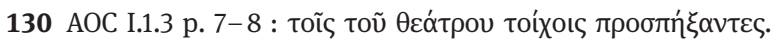

131 Thdt. h.e. 2.17.5. Zu dieser Episode siehe auch MATTHEIs (2013) 227-230.

132 CAMERON (1976) 292. Ein weiteres Beispiel sind Unruhen im Hippodrom von Konstantinopel zugunsten des abgesetzten Patriarchen Euphemius im Jahr 496, siehe GrEatrex (1997) 64 Anm. 22. 133 Z. B. der Streit um die Formel des Trisagion, siehe Jo. Mal. chron. 16.19(407-408); HeUCKE (1994) 282-285; Greatrex (1997) 64; MEIER (2007) 232. Allerdings ist der Zusammenhang zwischen Zirkusparteien und religiöser Parteinahme ofmals unklar, vgl. CAMERON (1976) 130, 242-243, 292. Auch bei der Agitation gegen den Bischof von Edessa griffen kirchliche Parteien im Jahr 588 auf die Hilfe

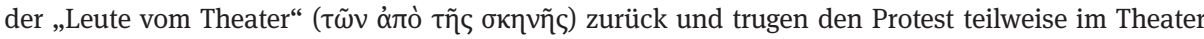
vor, dazu Evagr. h.e. 6.7 (FC 57,2). Weitere Beispiele für kirchliche Claqueure siehe bei LIEBESCHUETZ (1972) 217.

134 Coll. Avell. 1.6-7 (= CSEL 35.1, 2-3); Soz. h.e. 7.15.13-14. Text siehe Anm. 35 Kapitel VI. 135 Siehe Anm. 391-393 Kapitel V. 
wie Kreuze oder Reliquien mitunter auch in Spielstätten aufgestellt wurden und in Ausnahmefällen dort sogar die Liturgie gefeiert werden konnte. ${ }^{136}$

Eine Episode aus der Vita des Mönchvaters Hilarion verdeutlicht, wie in der hagiographischen Propaganda das Zirkuswesen sogar in positiver Weise als ein christliches Medium fungieren konnte: ${ }^{137}$ Hieronymus berichtet davon, dass in der Gegend von Gaza ein christlicher Magistrat ein Wagenrennen gegen die Pferde eines paganen Magistraten aus einer anderen Stadt organisierte. Als ihm zu Ohren gekommen war, dass der heidnische Konkurrent seine Pferde verflucht hatte, bat er Hilarion um Hilfe, da er sich aufgrund seiner Amtspflichten der Ausrichtung des Wagenrennens nicht entziehen konnte. Hilarion half ihm schließlich, indem er die Pferde, den Rennstall, die Wagenlenker und sogar die Startschranken segnete, und am Ende dieser Erzählung siegten erwartungsgemäß die christlichen Pferde. Eine solche Art der literarischen Darstellung hatte also nicht mehr die Trennung von Kirche und Spielen im Sinn, sondern konstruierte vielmehr eine nützliche Kollaboration zwischen beiden Bereichen, in denen der christliche Gott gegenüber den Heiden sein Wirken manifestierte. ${ }^{138}$

Hinsichtlich der Bühnenparodien auf das Christentum haben verschiedene Forscher bereits aufgezeigt, dass derartige Satiren in der Zeit nach dem 4. Jahrhundert nicht mehr unbedingt in einer feindlichen Haltung gegenüber Christen begründet lagen. Vielmehr war die Parodie eine allgemeine Eigenschaft des mimischen Genres und das Christentum stand als Gegenstand sozialer Karikatur sicherlich in einer Reihe mit anderen Themen, über die sich komische Schauspieler lustig machten. ${ }^{139}$ Die sogenannten Taufmimen, von denen zuvor die Rede war, hatten in ihrem literarischen Rückgriff auf die Zeit der Verfolgung die Intention, die Teilnehmerschaft an Schauspielen als ein feindliches Milieu zu konstruieren - jedoch zu einem Zeitpunkt, an dem ein solcher Gegensatz zwischen Christen und der übrigen Gesellschaft gar nicht mehr existierte. ${ }^{140}$ Theaterschaffende sollten durch derartige Geschichten zwar als „popular antitheses“ der Heiligen fungieren, ${ }^{141}$ allerdings implizieren spätere Zeugnisse von

136 Cod. Iust. 1.3.26 enthält ein Verbot derartiger Praktiken. Wie LANIADo (2002) durch eine Untersuchung der Scholien gezeigt hat, hatte anscheinend eine provisorische Messe im Theater von Emesa aufgrund eines vorherigen Erbebens den Anlass für diese Konstitution gegeben. Vgl. zudem Socr. h.e. 7.22.13-18 zu Litaneien im Hippodrom unter Theodosius II.

137 Hier. vita Hilar. 11.3-13.

138 Vgl. LUGARESI (2007) 34. Die von Johannes Malalas geschilderte Episode des samaritanischen Usurpators Julianos weist in eine ähnliche Richtung: Als bei einem Wagenrennen in seiner Anwesenheit ein christlicher Wagenlenker gewann, wurde dies als schlechtes Omen gedeutet und der Wagenlenker wurde ähnlich einem Märtyrer aufgrund seines Glaubens hingerichtet (Jo. Mal. chron. 18.35).

139 FRENCH (1985) 203-206; WEBB (2008) 126-127. Mimenschauspieler machten sich auch über jüdische Praktiken lustig, vgl. WeISMANN (1975) 48; WeISs (1996) 446.

140 LIM (2003) 97-98.

141 LIM (2003) 105. 
Bühnenparodien aus dem 5. und 6. Jahrhundert, dass inzwischen Christen selbst an der Karikatur ihrer eigenen Kirche beteiligt gewesen sein müssen. ${ }^{142}$

Auch die Trennung der Kirche von den Spielschaffenden, also ihre Exkommunikation, sollte trotz zahlreicher Bestimmungen nicht als absolut aufgefasst werden. In der eben geschilderten Episode des Hilarion konnte auch ein Christ Wagenrennen veranstalten, und andere Quellentexte implizieren gleichfalls die Existenz von Wagenlenkern, die Christen waren. ${ }^{43}$ Obwohl eine Exkommunikation nicht $\mathrm{zu}$ einem christlichen Begräbnis hätte führen dürfen, sind uns aus der römischen Basilika St. Paul vor den Mauern, den römischen Callixtus-Katakomben sowie Florenz und Aquileia Grabsteine eines Pantomimus, einer Schauspielerin und verschiedener Wagenlenker überliefert, die eine christliche Grabstätte erhielten. ${ }^{144}$ Gleiches muss für den umstrittenen Epitaph eines auriga aus der christlichen Nekropole von Mérida gelten [Abb. 1]. ${ }^{145}$ Während der Vandalenverfolgung erlitt der archimimus Masculas für die Standhaftigkeit seines katholischen Glaubens sogar das Martyrium, ohne dass in der Beschreibung durch Victor de Vita irgendwo erwähnt ist, dass er seinen Beruf aufgegeben habe. ${ }^{146}$ In der Praxis scheinen Spielschaffende also weiterhin ihren

142 Siehe Anm. 32-33 Kapitel II; WebB (2008) 127; Vogt (1931) 269.

143 Jo. Mal. chron. 18.35(446); Vit. Aux. 33 (= PG 114, 1401).

144 ILCV I 578 = CIL 10116: pantomimus; ILCV I 586 = CIL XI 1730: actrix c(onsularis?) domus; ILCV I 570 = CIL VI 10066 = ILS 5303: aurica = auriga? (439 n. Chr.); ICUR 5688 = SEG XXXII 1065 = SEG XLI

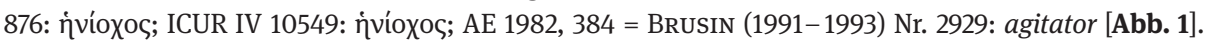
Für einen christlichen Zusammenhang sprechen sowohl das Formular der Inschriften als auch die Fundorte. Ein weiterer Epitaph aus Rom dürfte dieser Liste hinzuzufügen sein (ILCV 574 = CIL VI 10079). Die auf ihm verzeichneten Namen Constans, Barbarus und Cermarus können aufgrund von Illustrationen auf dem Grabstein mit einem Wagenlenker und seinen beiden Rennpferden identifiziert werden; der Fundort in der Domitilla-Katakombe verweist auf einen christlichen Hintergrund. Auch der Wagenlenker Liber könnte aufgrund eines Medaillons mit christlicher Symbolik [Abb. 57] und einer Inschrift aus einer Katakombe als Christ identizifiert werden, vgl. FERRUA (1947) = FERRUA (1991) 197-206. In Nordafrika stiftete ein actor Ingenuus für seinen christlichen Freund einen Grabstein und könnte demnach selber Christ gewesen sein (ILCV I 585 = CIL VIII $25817=$ AE 1978, 880) [Abb. 143]. Für weitere Beispiele solcher christlichen Akteure des Spielewesens siehe CusciTo (1994) 107-128; JimÉnEz SÁNCHEz (2006b) 81-83; CiCU (2012) 70-71.

145 Der Epitaph eines Sabinianus auriga aus Mérida wurde aufgrund paläographischer Merkmale, des Formulars sowie des archäologischen Kontexts auf das späte 4. bis 6. Jdt. datiert; siehe Edition in Ceballos Hornero (2002) Nr. 76 und Abbildung in ARce (2001) 273. Dieser Umstand ließ ARCE (2001) 273-277 vermuten, dass der Ausdruck auriga vielmehr metaphorisch zu verstehen sei und sich nicht auf einen tatsächlichen Wagenlenker beziehen könne. Dieser Fall liefert insofern ein treffendes Beispiel dafür, wie die offizielle Ablehnung der Spiele in den patristischen Quellen selbst von modernen Forschern als realistisch angesehen wird. Da in Mérida eine Nutzung des Zirkus bis zu Beginn des 5. Jdts. anzunehmen ist (Anm. 189 Kapitel IV) und Mosaikfunde die Popularität von Wagenrennen bezeugen (siehe Kapitel V „Aristokratische Bilderwelten“), erscheint - verbunden mit den zuvor genannten Zeugnissen aus Rom - die Grablege eines Wagenlenkers auf dem christlichen Friedhof in Mérida allerdings plausibel. Die Zweifel an der Identität von Sabinianus sind somit unbegründet, vgl. ebenso García Moreno (2001) 14; Jiménez SÁnchez (2006a) 106; Nogales Basarrate (2008) 188. 146 Vict. Vit. 1.47 (ed. Vössing 2011). 
christlichen Glauben praktiziert zu haben, ohne zwangsläufig von der kirchlichen Communio ausgeschlossen zu werden. ${ }^{147}$

\section{Agonale Metaphorik}

Zuletzt soll auf eine linguistische Überlagerung beider Bereiche verwiesen werden, nämlich die reiche Nutzung von Metaphern aus der Welt der Spiele und der Agonistik. Verurteilten die Kirchenväter zum einen Spiele als verwerflich und sündhaft, scheuten sie jedoch nicht davor zurück, in ihrer bildlichen Sprache erstaunlich detailgetreu auf die Welt der Spiele zu rekurrieren, um somit alternative spectacula zu konstruieren. ${ }^{148}$ In der Forschung ist vor allem die agonistische Sprachwahl ausführlich analysiert worden: ${ }^{149}$ Ein häufiges Motiv ist hierbei Gott als agonothetes, welcher den Agon auf Erden, d. h. das Lebenszeugnis eines Christen gegenüber den Versuchungen, beurteilt und belohnt. ${ }^{150}$ Anstelle eines gewöhnlichen Athleten im physischen Wettkampf sollte man zu einem wahren athleta Christi werden und so der Welt eine Auseinandersetzung geistiger Natur bieten. ${ }^{151}$

Eine andere, weitverbreitete Metapher findet sich in der Rede vom spectaculum deo, dem Schauspiel für Gott (und vor den Menschen). ${ }^{152}$ In Anlehnung an den 1. Korintherbrief ${ }^{153}$ stellten zahlreiche Kirchenväter den weltlichen spectacula eine ei-

147 Ein weiteres Zeugnis sind zwei kommemorative Inschriften in den heiligen Thermen von Gadara aus dem 5. Jdt., die durch einen Pantomimen (SEG XLVII 1990 = Di SEGNI (1997) Nr. 6: ópXıтŕৎ) und

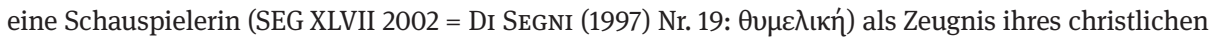
Glaubens hinterlassen wurden.

148 WEBB (2008) 205: „a binary opposition between an improving spectacle and the corrupting images of the stage“.

149 Lugaresi (2003a); Jiménez SÁnchez (2000); PApathomas (1997); Stewart (1984); Cameron (1976) 215-216; Merkelbach (1975); Weismann (1972) 111-121. Die Metapher des Agons beruht auf 1. Kor. 9.24-27; siehe auch SPICQ (1937). So wie Paulus' agonistische Metapher den antiken Wettkampf zum Vorbild nahm, geht im übrigen auch seine Metapher vom „guten Lauf“ im Stadion des Lebens (Tim. 4.7; Röm. 9.16) auf antike Exempla zurück, vgl. Pi. N. 6.6-7; Ov. Trist. 1.9.1; LATTimore (1942) 169. Zudem war das Bild des Athleten zu Beginn der Spätantike anscheinend ein weitverbreitetes rhetorisches Motiv, hierzu REMIJSEN (2011) 169-176. Zu „urbanem“ Vokabular in asketischen Beschreibungen siehe SARADI (2006) 102-105.

150 Ast. Am. hom. 9.3 (ed. Datema 1970); Bas. Sel. or. 27.1 (= PG 85, 309); Chrys. de in. glor. 1 (ed. Malingrey 1972, SC 188); Ioh. Apht. vit. Sev. (= PO 2, 219-220); Sev. Ant. hymn. 269 (= ALLENHAYWARD (2004) 172-73).

151 Paul. Nol. epist. 24.9, 40.10; Chrys. hom. 15 in Phil. 5 (= PG 62, 294-295); Sev. Ant. hom. cat. 91 (= PO 25.1, 7-27); Thdt. h.e 4.22.19-20. Ein Mönchsroman des 5. Jdts. vom Sinai gebraucht derart detaillierte Metaphern des Ringkampfes, dass man beim Verfasser eine eigene Kenntnis solcher Wettkämpfe voraussetzen muss (Ps.-Nil. narr. 3.12-13). Ähnlich detalliert ist auch Severus v. Antiochia in seiner Gegenrede zu den Olympischen Spielen, vgl. Sev. Ant. hom. cat. 94 (= PO 25.1, 71-74). 152 Dazu LugaRESI (1998) 687-692.

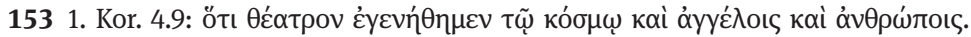


gene Alternative gegenüber, nämlich die divina spectacula, ${ }^{154}$ welche spiritueller Natur seien und entweder in christlichen Werken der Nächstenliebe ${ }^{155}$ oder in den Taten Gottes und der Märtyrer bestünden. ${ }^{156}$ Die Arena der guten Werke sei der wahre Ort, an dem es galt, sich als Schauspieler auszuzeichnen, ,wobei Gott selbst der Zuschauer ist“ ${ }^{157}$ Wie Lucy Grig und Leonardo Lugaresi in ihren Studien herausgearbeitet haben, übernahm gerade das dem neuen Testament entlehnte Bild des Märtyrers in der christlichen Propaganda der ersten drei Jahrhunderte klassische dramatische Elemente. Es diente somit auf der literarischen Ebene und im Rahmen von Märtyrerfeiern als ein alternativer, jedoch zugleich vom Theater inspirierter Gegenentwurf. ${ }^{158}$ Eine ähnliche Tendenz ist bei jüdischen Autoritäten zu beobachten, die in ihre Predigten ebenfalls Begriffe und Bilder aus der Welt der Spiele integrierten. ${ }^{159}$

Auch im Bereich der Ikonographie wurde eine Distanzierung vom antiken Spielewesen nicht konsequent verfolgt. Stattdessen konnten gewisse Bildmotive, mit denen keine spezifisch pagane Symbolik verbunden war, durchaus in christliche Kunstwerke integriert werden. So wurde der Topos des Agons nicht nur in literarischen Werken verwendet, sondern das Motiv des Siegeskranzes fand auch Eingang in die christliche Kunst. ${ }^{160}$ Darstellungen siegreicher Rennpferde, die aufgrund ihrer Assoziation mit dem Zirkusgeschehen eine Semantik von Triumph und Erfolg vermittelten, konnten als ikonographische Motive in christlichen Grabkontexten verwendet werden und als Symbole des Lebenslaufs fungieren [Abb. 3-4]. ${ }^{161}$ Der Prophet Elias wurde als auriga spiritualis bezeichnet und in der Figur eines Rennfahrers dargestellt, der auf seinem Wagen gen Himmel fuhr [Abb. 5]. ${ }^{162}$ Eine weitere Übernahme lässt sich in der Ikonographie des thronenden Christus erkennen, die zum Teil auf die Darstellung des Kaisers bei Spielen Bezug nahm. Sicherlich stand bei der Adaption die herrschaftliche Semantik dieses Bildtypus im Vordergrund, dennoch verwendete man als Vorbild eine Bildsprache, die unter anderem auf den Kaiser im Geschehen des Hippodroms Bezug nahm. ${ }^{163}$ Christliche Künstler griffen demnach bewusst auf ein Ereignis zurück, das in der spätantiken Gesellschaft eine zentrale Bedeutung besaß und dessen allgemeine

154 Aug. enarr. psalm. 80.23 (= PL 37, 1040).

155 Die metaphorisch durchkomponierte Abhandlung eines solchen alternativen spectaculum findet sich bei Paul. Nol. epist. 13.12-16; siehe hierzu SchlaPBACH (2013).

156 Weismann (1972) 105-110; Aug. serm. 51.2 (= PL 38, 333-334); Aug. serm. Den. 17.7 (ed. Morin, Misc. Agost. 1930 I); Quodv. symb. 1.2.2-5 (= CC 60, 307); Ast. Am. hom. 3.1.2 u. 10.6 (ed. Datema 1970); Chrys. pan. mart. 11 (= PG 50, 645-647); Chrys. hom. 10 in Col. 4 (= PG 62, 370 - 371); Jac. Ser. hom. de spect. 4 (= Moss (1935) 107).

157 Paul. Nol. epist. 13.16: deo ipso spectatore.

158 GRIG (2004) 34-47; LUGARESI (2008) 320 - 345.

159 WEISS (2010) 637.

160 Mittag (1999) 173 Anm. 8; Malineau (2006) 189.

161 Dunbabin (1978) 103; Blázquez Martínez (1986) 62-63; KlumbaCH (1952) Nr. 25 -26.

162 Quodv. symb. 1.2.8 = CC 60, 308. Zu dieser Ikonographie siehe LANDESMANN (2004) 81-217. 163 GRABAR (1971) 192-195. L'ORANGE (1965) 99-103 führt die Darstellung des Theodosiusobelisken als Beispiel an. Siehe auch HARLEy (2009) 319. 
Bekanntheit sich gut zur bildlichen Adaption eignete, auch wenn ein Zusammenang der offiziellen Position nach eigentlich zu vermeiden war.

Die kirchlichen Autoritäten der Spätantike bedienten sich also an zahlreichen Stellen literarischer und bildlicher Metaphern, die dem Spielewesen entnommen waren. Zweck dieser Sprachwahl war es sicherlich, eine katechetische Aussage in der Lebenswelt der Zuhörer zu verorten; ${ }^{164}$ gleichzeitig sollten aber auch auf sprachlicher Ebene zentrale Begriffe dieser „verwerflichen“ Welt umgedeutet werden, um ihnen eine christliche Interpretation gegenüberzustellen. So verurteilte Quodvultdeus nicht den Wunsch des Zuschauens (voluntas spectandi) an sich, nur sollte sich die Aufmerksamkeit eines Christen anstelle von Schauspielern auf Figuren der Bibel und sittliche Vorbilder richten. ${ }^{165}$ Seiner Meinung nach besäßen die Menschen zwei Maßstäbe, um spectacula zu erfassen, nämlich eine fleischlich-weltliche und eine spirituelle Dimension. ${ }^{166}$ Anhand der letzteren Blickweise könne das Schicksal eines Märtyrers durchaus als wahrhaftes spectaculum begriffen werden.

Gleichwohl weltliche und geistige spectacula daher auf den ersten Blick einen ähnlichen semiotischen Charakter aufwiesen, war von einer solchen Strategie aber kein großer Erfolg zu erwarten, da beide Arten dramatischer Inszenierung auf verschiedenen Ebenen stattfanden. Die Semantik des herkömmlichen spectaculum bezog sich auf ein soziales Ereignis der Unterhaltung, wohingegen die von den Kirchenvätern angestrebte Umdeutung einen Diskurs im Sinn hatte, der auf einer spirituell-theologischen Ebene angesiedelt war. ${ }^{167}$ Es ist nicht verwunderlich, dass Gläubige Johannes Chrysostomus vorhielten, dass Zirkusspiele eine andere Freude verursachen würden, als sie der Bischof im Gottesdienst sah. ${ }^{168}$ Ein spectaculum der Märtyrer oder das Leben als ein christlicher Agon stellten ideelle Kategorien auf, deren spirituelle Funktion jedoch vollkommen anders und daher nicht vergleichbar mit den physischen und emotionalen Aspekten einer Spielveranstaltung war - geschweige denn mit der auf einer sozialen Ebene angesiedelten Funktion von Spielen als Unterhaltung. ${ }^{169}$ Es darf

164 Interessanterweise findet sich eine ähnliche Verwendung bei rabbinischen Unterweisungen, dazu WeIss (1996) 445.

165 Quodv. symb. 1.2.10 (= CC 60, 308). Ähnlich auch Augustinus, vgl. LugARESI (1998) 586-602. Zur bildlichen Realisierung dieser christlichen voluptas spectandi in Märtyrerdarstellungen siehe SALOMONSON (1979). Insofern hätte ein moralisch „gutes“ Theater eigentlich existieren können, allerdings haben die Kirchenväter diese alternative Option niemals weiter diskutiert.

166 Aug. serm. 51.2 (= PL 38, 333): aliter spectant carnales, aliter spirituales. Ebenso spricht Sev. Ant. hom. cat. 94 von „spectacles des luttes spirituelles“ (übers. in PO 25.1, 73). Schon Cicero formulierte ein ganz ähnliches Bild, indem er sinnliche und geistige voluptates voneinander unterschied: laetitiam in animo, commotionem suavem iucunditatis in corpore. (Cic. fin. 2.13).

167 LUGARESI (1998) 677-685.

168 Vgl. Chrys. Anna 42 (PG 54, 661-662); dazu die Analyse von PASQUATo (1976) 325-331 über das theatrum spirituale. Ebenso Chrys. hom. 18 in Jo. 4 (= PG 59, 119-120).

169 Die Bezeichnung des Propheten Elias als spiritualis auriga ist ein Beispiel für die spirituelle Umdeutung herkömmlicher spectacula (Quodv. symb. 1.2.8 = CC 60, 308). In einem anderen Vergleich assoziiert Augustinus die harmonischen Bewegungen eines Pantomimen mit der Harmonie der Sitten, die man durch den inneren Tanz nach dem Klang des Sängers Christus erreichen könne - man solle 
auch bezweifelt werden, ob die von Kirchenvätern komponierten Hymnen als Antwort auf populäre Gesänge des Theaters einen tatsächlichen Erfolg aufweisen konnten. ${ }^{170}$ Das eher der Askese entstammende, allegorische Denkkonzept konnte die bestehende gesellschaftliche Praxis nicht ersetzen, die in ihrer Funktion des allgemeinen Vergnügens geschätzt wurde und daher einen anderen sozialen Wert repräsentierte.

In Bezug auf die Metapher des Märtyrers als spectaculum ist zudem anzumerken, dass die Kirchenväter ab dem 4. Jahrhundert erstaunlich selten das an sich naheliegende Argument des Martyriums gegen die Spiele vorbrachten. Schließlich hatten die Spielräume in der Vergangenheit den Hintergrund für Verfolgungen und Hinrichtungen geliefert, waren also aufgrund der erlittenen Opfer als gleichsam „feindliche Räume“ abzulehnen. Wie oben dargelegt wurde, bezogen sich die Kritikpunkte an den Arenakämpfen jedoch vielmehr auf die innerliche Disposition der Zuschauer. ${ }^{171}$ Dass ein Zusammenhang mit den Martyrien nur selten vorgebracht wurde, mag daher der Tatsache geschuldet sein, dass wir es auch in diesem Fall mit einer Instrumentalisierung der Spielräume durch die christlichen Autoren zu tun haben. Obwohl man für eine Schließung der Amphitheater plädierte, stellten sie in der spätantiken Propaganda wiederum den perfekten Ort dar, um das „Spektakel“ eines christlichen Martyriums zu verdeutlichen: „la simbolizzazione ludica che per i cristiani assume il loro martirio.“172 Der Märtyrer konnte gleichsam nur an diesem Ort zu einem zweiten Arenakämpfer werden. Auch die als literarische Gegenbilder konzipierten Taufmimen

nicht mit dem Körper, sondern mit dem Geist tanzen (Aug. serm. 311.4 .7 = PL 38, 1416): non corpore sed mente saltantem. Eine weitere Metapher war dem Zirkuswesen entlehnt und bezog sich auf den Wagenlenker mit seinen vier Pferden. Dieses Bild stellte entweder die vier Elemente unter der Seele als harmonische Lenkerin dar (Tert. anim. 53.3; Potam. tract. 1.45-50) oder die vier essentiellen Tugenden eines Christen auf seinem Weg zum Himmel (Caes. Arel. serm. 42.1). Auch Gregor Thaumaturgos wurde mit einem Wagenlenker verglichen, der alles unter das Joch des Glaubens stellte (Gr. Nyss. v. Gr. Thaum. = PG 46, 954B), dazu WeISmanN (1972) 185. Andere Metaphern verwendeten die Welt der venationes als Vorbild, vgl. Chrys. theatr. 4 (= PG 56, 268-270); Pall. $h$. Laus. 54. So wurde der Sieg über wilde Tiere und das Biest auch zu einem Bild des Christentums, vgl. WiedemanN (1992) $154-155$.

170 Severus von Antiochia komponierte z. B. eigene christliche Hymnen als Antwort auf die Popularität von Bühnenliedern unter den Einwohnern Antiochias, vgl. Ioh. Apht. vit. Sev. (= PO 2, 244); Brooks (1911); Allen-HAYwARD (2004) 54-55. Zu den Hymnen in Mailand unter Ambrosius siehe Aug. conf. 9.7; DudDEN (1935) 295-96; FonTAINE ET AL. (1992) 16-19. Zu Johannes Chrysostomus siehe Chrys. educ. lib. 34 (ed. Malingrey 1972, SC 188); Liebeschuetz (2011b) 208. Vgl. auch Caes. Arel. serm. 6.3, 75.1; Cassiod. in psalm. 39.6. Zwar sollten kirchliche Hymnen durch ihre Kommunikationsform ebenfalls breitere Schichten erreichen, dennoch besaßen sie zunächst eine didaktischtheologische und keine unterhaltende Funktion, vgl. WECKWERTH (2010). Erst das religiöse Theater des Mittelalters konnte eine ähnliche Alternative bieten, wenngleich auch hier die didaktische Funktion im Vordergrund stand und lediglich dramatische Formen übernommen wurden, dazu Goody (1997) 101; HARdison (1965) 15-20, 284-288; FLANIGAN (1991); PUCHNER (2002) 322-323. Vgl. auch SchnuSENBERG (1981) 57-93 zu Funktion und Charakter der frühchristlichen Liturgie.

171 Anm. 20 Kapitel II.

172 CARFora (2009) 112. Zu diesem Thema siehe auch PotTER (1993); VAN DEN HoECK-HERRMANN JR. (2001). 
bringen dies deutlich zum Ausdruck: Zwar verneinte man den Umgang mit dem sündigen Theater, zugleich bot es aber die perfekte Bühne für ein Bekehrungsparadigma. ${ }^{173}$

\section{Fazit: Zwischen Anspruch und Wirklichkeit}

Zusammenfassend ist festzuhalten, dass die kirchlichen Argumentationsstrategien in der Praxis keine konsequente Dichotomie zwischen Christentum und römischen Spielen zu schaffen vermochten. Obwohl durch den literarischen Diskurs eine solche Realität unter den Gläubigen erzeugt werden sollte, ${ }^{174}$ scheiterte dieses Vorhaben an verschiedenen Faktoren: Zunächst wies der literarische Diskurs selbst Ambivalenzen und Stereotypien auf. Viele der vorgetragenen Denkkonzepte und Argumente fußten auf älteren Ideen, insbesondere der Argumentation Tertullians, so dass sie auf die Adressaten stereotyp und in diesem Sinne unzeitgemäß gewirkt haben müssen. ${ }^{175}$ Ebenso flossen Konzepte in die Konstruktion dieser Dichotomie ein, die einer asketischen und äußerst moralisierenden Weltvorstellung entstammten und damit kaum die Lebenswirklichkeit des Bewohners einer in antiken Traditionen verwurzelten Stadt abbildeten. Der Gegensatz zu klassischen Kulturformen entstammte eigentlich einer Zeit, in der sich die Christen noch von der übrigen Welt hatten absetzen müssen. ${ }^{176}$ Spätestens ab dem 5. Jahrhundert stellte aber das Christentum die Mehrheit, so dass die vormalige Argumentation dieser Tatsache ebenfalls in irgendeiner Weise hätte angepasst werden müssen. Vielmehr machte es der zunehmende Kontakt mit der allgemeinen Lebenswelt schließlich notwendig, sich auf der sprachlichen Ebene auf die Spiele einzulassen und Metaphern eines alternativen spectaculum zu schaffen. Bereits diese sprachlich nicht konsequent durchgehaltene Distanzierung konnte Probleme der Glaubwürdigkeit verursachen, wie Argumente der Gläubigen gegenüber Tertullian und Novatian zeigen, die den Besuch von Agonen mit einem Verweis auf Paulus' agonale Metaphern rechtfertigten. ${ }^{177}$ Zudem waren die konstruierten Alternativen auf einer geistigen Ebene angesiedelt und konnten somit nicht die soziale Funktion der Spiele als Unterhaltung ersetzen. Ebenso ist der durch die Förderung von Spielen zu erzielende Ruhm von einer anderen - und vielleicht oftmals unmittelbareren und attraktiveren - Natur gewesen als ein Engagement im Bereich kirchlicher Caritas. Zwar zielte der vorgebrachte Diskurs auf eine profunde Christianisierung der

173 Siehe Anm. 158 Kapitel II.

174 Vgl. Lim (2009) 501; LANDWEHR (2009) 106.

175 In seiner Analyse der Predigten Jacobs v. Serugh erkannte Moss (1935) 93 bereits die Ähnlichkeit zu Diskursen des 2. und 3. Jdts. und nahm daher die Existenz von „drawn up manuals“ an, die für derartige Kontroversen benutzt worden seien. Zwar geht eine solche Annahme zu weit, sie verweist aber auf die problematische Stereotypie dieser Texte.

176 Vgl. MARKus (1993) 101.

177 Ps. Cypr. [Nov.] spect. 2 (= CSEL 3.3, 4); Tert. spect. 18. 
versammelten Gemeinde ab, jedoch wurde der Kaiser als weltliches Haupt bewusst davon ausgenommen. Um allerdings den eigenen Interessen einen wirklichen Nachdruck zu verleihen, hätte die Führungsperson des gesellschaftlichen Handelns miteingeschlossen werden müssen.

Auch fern der sprachlichen Ebene, d.h. auf dem Gebiet der lebensweltlichen Praxis, sind Ambivalenzen und Brüche zu konstatieren: Eine Stigmatisierung der Spiele ließ sich nicht einmal innerhalb des eigenen Klerus realisieren, der offensichtlich häufiger die konstruierte Grenze überschritt, als es die Schriften der Kirchenväter Glauben machen. Es muss davon ausgegangen werden, dass zahlreiche Kollegen im priesterlichen oder bischöflichen Amt den Forderungen eines Johannes Chrysostomus, Augustinus oder Severus von Antiochia indifferent gegenüberstanden und in der seelsorgerischen Praxis den Besuch von Spielen durchaus tolerant handhabten. ${ }^{178}$ Zuletzt hielten sich christliche Symbolik und innerkirchliche Politik nicht von den Räumen des Spielewesens fern, sondern sowohl die Gebäude in ihrer Funktion als öffentliche Versammlungsräume wie auch das performative Geschehen konnten für religiöse Belange in Anspruch genommen werden.

Alle diese Faktoren führen zu dem Ergebnis, dass es der patristische Diskurs mit seinen Argumenten nur schwerlich vermocht hat, eine wirkliche Änderung von Lebensgewohnheiten herbeizuführen. In zu vielen Punkten besaß die konstruierte Dichotomie zwischen christlichem Lebenswandel und weltlichem Vergnügen argumentative Schwächen oder wurde durch die Praxis kirchlicher Verantwortungsträger ad absurdum geführt. Auch wenn die spätantike Literatur augenscheinlich von einem Kampf der kirchlichen Autoritäten gegen die Spiele geprägt ist, wird bei einer genaueren Analyse ersichtlich, dass diese auf der moralischen Ebene geführte Auseinandersetzung ein $\mathrm{zu}$ schwaches Fundament besaß, um einen faktischen gesellschaftlichen Wandel in diesem Lebensbereich herbeizuführen. Vor allem orientierte sich der Diskurs in vielen Aspekten an einer Zeit, in der das Christentum noch keine offizielle Religion gewesen oder sogar verfolgt worden war, so dass ein Gegensatz zum sozialen Umfeld wie der Welt der Spiele leichter konstruiert werden konnte. Ab dem 4. Jahrhundert allerdings war eine solche Dichotomie immer weniger aufrechtzuerhalten.

Es stellt sich angesichts der Dekonstruktion der christlichen Kritik letztlich die Frage, warum die kirchlichen Autoritäten ihren Kampf gegen die Spiele so beharrlich weiterverfolgten - anders formuliert: War es wirklich die komische Darstellung eines Mimenschauspielers, die einen fortwährenden Widerstand hervorrief? Oder stellte

178 Vgl. BRown (1988) 319-320. Choricius v. Gaza und Isidor v. Pelusium sind Beispiele für alternative Positionen. Obwohl beide Christen waren, verteidigte Choricius Mimenschauspiele und plädierte für eine pragmatische, nicht von moralischen Verurteilungen dominierte Einstellung; siehe Chor. Apol. mim.; WeBB (2002) 299-300. Isidor wiederum analysierte, obgleich Bischof, die Spiele nicht mit christlichen Argumenten, sondern versuchte auf sophistische Weise, ihnen die Tugendhaftigkeit des Bürgers gegenüber zu stellen; siehe Isid. Pel. epp. 5.185(1469) (ed. Évieux 2000, SC 454); LIM (1997a) 68 u. 72. 
dies vielmehr ein strategisches Argument dar, während unter den zahlreichen in diesem Kapitel vorgestellten Konflikten einige Felder als fundamentaler zu betrachten sind? Studien der vergangenen Jahre von Ruth Webb, Robert Markus und Alan Cameron scheinen in diesem Zusammenhang eine mögliche Antwort aufzuzeigen, nämlich die Bedeutung klassischer Schauspiele für die Herausbildung einer spezifisch christlichen Identität. Nicht nur in tertullianischer Zeit, sondern auch während der folgenden Jahrhunderte ging es darum, einerseits innerhalb der Kirche einen festen Zusammenhalt zu finden, nach außen hin diese Einigkeit aber auch durch ein gemeinsames Feindbild zu erzielen, nämlich eine konsequente Distanzierung zur traditionellen Festkultur. ${ }^{179}$ So lässt sich in der kirchlichen Position des 5. und 6. Jahrhunderts die Tendenz beobachten, keine „neutrale“ Sphäre mehr zu akzeptieren, sondern jede Handlung, die nicht als spezifisch christlich interpretiert werden konnte, nun als pagan anzusehen. ${ }^{180}$ Anstelle einer möglichen Kompromissfindung verstärkte sich also die Dichotomie zur klassischen Kultur noch weiter. Warum ausgerechnet die Spiele in diesem Themenfeld als prominentes Feindbild hervortraten, lässt sich durch ihre Dominanz im öffentlichen Raum erklären. Der Versuch kirchlicher Autoren, eine alternative Stadtgesellschaft zu konstruieren, wurde bereits zuvor thematisiert. Hier ging es letztlich um den Wunsch, den Lebensrhythmus der eigenen Gläubigen ausreichend zu kontrollieren: welche Prioritäten sie ihrer Freizeitgestaltung gaben und welche sozialen Werte sie welcher Institution zuschrieben. Wenn man als Seelsorger nachhaltigen Erfolg haben wollte, musste man die Gläubigen trotz der Konkurrenz anderer Vergnügungen für einen Besuch des eigenen Gottesdienstes gewinnen. Der Widerstand gegen mimetische Darstellungen mag als Versuch gedeutet werden, selbst die hinter dem Theater stehende „symbolische Realität“181 einer Kontrolle zu unterwerfen.

Mit der Frage nach den sozialen Prioritäten waren nicht zuletzt finanzielle Interessen verbunden. Zwar sind die exakten Kosten für spätantike Spiele nur selten überliefert, aber eine Inschrift aus Caesarea Maritima bezeugt für das späte 5. Jahrhundert eine jährliche Zahlung von circa 700 solidi für den Unterhalt der Pferdezucht

\footnotetext{
179 Vgl. Anm. 44 Kapitel II; WEBB (2008) 198-199: „In many ways the theater provided a perfect foil against which the new institution of the Church could define itself [...] Much of the Christian discourse about the theatre may not be primarily about the theater at all, but may serve instead as a way of discussing other problems within the Church and in the Church's relation to society and to individuals“. Vgl. auch die Analyse von Richard Lim zur innerkirchlichen Funktion der Taufmimen (Anm. 38 Kapitel II). MARKUS (1993) 101: „Christians had to guard against the threat to their identity by becoming too assimilated [...] to dissociate themselves from the calendar of traditional Roman public festivals, and the celebrations, circus games, banquets, shows in the theatre and the hippodrome, associated with many of them".

180 CAMERon (2011) 789: „It is not so much a question of its „tenacity“, as a changing, far more comprehensive definition of paganism, a definition that eventually came to include any custom of practice not demonstrably Christian“. Vgl. auch MARKus (1991) 269-271 und MARKUS (1985) über den Unterschied zwischen den Zeiten Augustins und Gregors des Großen.
}

181 Vgl. BeEman (1993) 379. 
des lokalen Hippodroms. ${ }^{182} \mathrm{Zu}$ Justinians Zeiten wurden dem Statthalter von Ägypten 320 solidi für den Kauf von 36 Pferden zu Verfügung gestellt. ${ }^{183}$ Das monatliche Gehalt von zwei Startern des Hippodroms in Oxyrhynchus belief sich im Jahr 618 auf ungefähr 10 keratia, was einem Jahresgehalt von etwa 10 solidi entsprechen würde. ${ }^{184} \mathrm{Im}$ Vergleich dazu betrugen die Kosten für kleine Kirchenbauten, wie sie im Osten zwischen dem 5. und 6. Jahrhundert errichtet wurden, in der Regel zwischen 400 und 700 solidi. ${ }^{185}$ Diese beispielhafte Gegenrechnung mag verdeutlichen, welche finanzielle Dimension sich hinter der Förderung von Spielen verbarg und warum der Kampf um die Gunst der Gläubigen in dieser Sache so wichtig war.

Schließlich dürfte eine fundamentale Ursache für die radikale Ablehnung der Spiele in der persönlichen Glaubensüberzeugung der Kirchenväter selbst zu suchen sein, da bereits Aussagen des Neuen Testaments eine ähnliche Radikalität enthielten. ${ }^{186}$ Die bewusste Übernahme älterer Topoi lässt sich somit auch als Ausdruck einer genuinen inneren Überzeugung ansehen, dass man den Gläubigen die allein richtigen Lebensmodelle vermittelte. ${ }^{187}$ Die vorliegende Untersuchung hat allerdings aufgezeigt, wie eine vollkommene Radikalität und Pauschalisierung bei näherer Analyse zu Inkonsequenzen und Implausibilitäten führen musste. Die dem kirchliche Diskurs innewohnenden Ambivalenzen erklären dessen Scheitern, was eine Kontinuität der Spiele vor allem an den Orten möglich machte, wo sich die kirchliche Umgestaltung urbanen Lebens von vornherein nicht vollständig durchsetzen konnte und Lebensvollzüge noch für eine lange Zeit durch traditionelle Eliten und herkömmliche Unterhaltungsgenres geprägt wurden. ${ }^{188}$ Auch fand die kirchliche Position keine Unterstützung durch die politischen Maßnahmen der Kaiser, die sich trotz ihrer Zugehörigkeit zum Christentum weiterhin einem klassischen Herrscherbild verpflichtet sahen. Von ihnen wird deshalb im folgenden Kapitel die Rede sein.

182 LIfSHITZ (1957) 121 zu SEG XXX 1620. Zwar enthält die Inschrift eine jährliche Gesamtsumme von 5629 solidi, diese kann aber schwerlich nur für die Unterhaltung des Hippodroms aufgewendet worden sein. Eine Summe der einzeln aufgeführten Beiträge kommt auf ca. 713 solidi und ist als realistischer einzustufen, vgl. LANIADo (2002) 97.

183 Just. Edict. $13.15-16$.

184 P. Oxy. I 152.

185 Siehe HaEnsch (2006) 48.

186 Z. B. Matt. 5.30 oder 1. Kor. 10.21 (siehe Anm. 43 Kapitel II).

187 Vgl. Liebeschuetz (2011a) 321-322.

188 So ist es z. B. bezeichnend, dass sich im Osten allem Anschein nach die städtischen Eliten nicht signifikant an der Bautätigkeit von Kirchen beteiligten, also kein automatischer Transfer früherer euergetischer Aktivitäten in den kirchlichen Bereich erfolgte, dazu HAENsch (2006) 52. 